Article

\title{
Impact of Shale Anisotropy on Seismic Wavefield
}

\author{
Han $\mathrm{Li}^{1,2,3,4}$ (D) Xiwu Liu ${ }^{1,2}$, Xu Chang ${ }^{3,4, *}$, Ruyue $\mathrm{Wu}^{3,4}$ and Jiong Liu ${ }^{1,2}$ \\ 1 State Energy Center for Shale Oil Research and Development, Sinopec, Beijing 100083, China; \\ lihan@mail.iggcas.ac.cn (H.L.); liuxw.syky@sinopec.com (X.L.); liujiong.syky@sinopec.com (J.L.) \\ 2 State Key Laboratory of Shale Oil and Gas Enrichment Mechanisms and Effective Development, \\ Beijing 100083, China \\ 3 Key Laboratory of Shale Gas and Geoengineering, Institute of Geology and Geophysics, Chinese Academy \\ of Sciences, Beijing 100029, China; wuruyue@mail.iggcas.ac.cn \\ 4 Innovation Academy for Earth Sciences, Chinese Academy of Sciences, Beijing 100029, China \\ * Correspondence: changxu@mail.iggcas.ac.cn; Tel.: +86-10-8299-8281
}

Received: 21 October 2019; Accepted: 18 November 2019; Published: 20 November 2019

check for updates

\begin{abstract}
During unconventional resources exploration, ignoring shale anisotropy may lead to wrong seismic interpretations, thus affecting the accuracy and credibility of sweet spots prediction and reservoir characterization. In order to investigate the impact of shale anisotropy on the seismic wavefield, we propose a quantitative evaluation method by calculating the waveforms' amplitude and phase deviations. Based on the 3D elastic wave equation and the staggered-grid finite-difference method, the forward modeling theory with the consideration of shale anisotropy is established. Then, we use the envelope misfit (EM) and phase misfit (PM) parameters to illustrate the differences in waveforms' amplitude and phase morphology, which are caused by anisotropy. Lastly, by comparing the waveforms of the models with/without anisotropy and calculating their EM and PM values, a practical and quantitative evaluation method is constructed. We used synthetic models of different complexity and oilfield models to validate the proposed method. Through the research, we also gained some new insights about the anisotropy's effects. For a certain medium model, the impact of shale anisotropy on seismic wavefield is complicated and needs specific analysis. The proposed method provides a useful and quantitative tool for the evaluation of shale anisotropy's impact.
\end{abstract}

Keywords: shale reservoir; anisotropy; seismic wavefield; quantitative evaluation

\section{Introduction}

At present, more and more attention is paid on the research of unconventional resources exploration [1,2]. Unconventional resources, especially shale oil and gas, are realistic backup resources to meet the demand for fossil energy [3]. The shale oil and gas deposits are mainly stored in tight shale rocks or mud rocks. Shale is a sedimentary rock which is composed of fine debris, clay, and organic matter with a particle size of less than $0.0039 \mathrm{~mm}$ [4]. Rock physics research shows that shale has strong anisotropic characteristics (up to 70\%) [5,6] and most shale reservoirs currently developed have distinct seismic anisotropy [7-12].

Due to the strong seismic anisotropy of shales, seismic waves propagating through it will be influenced and changed. Therefore, if anisotropy is ignored, interpretations may lead to wrong models. Carcione [13] analyzed the influence of formation anisotropy on the amplitude of reflected seismic phases (PP and PS) and found that anisotropy of shale must be considered in AVO (amplitude variation with offset) analysis. Research in borehole microseismic monitoring for the hydraulic fracturing [14,15] concluded that failure to account for the shale anisotropy results in significant microseismic event location errors, and methods must be developed to circumvent the effects of anisotropy or to determine the anisotropy parameters. Tsvankin [16] reviewed state-of-the-art in modeling, processing, and 
inversion of seismic data for anisotropy media and concluded that the anisotropy has a strong influence on seismic data, especially for the shear and mode-converted wavefields. Through the forward modeling research based on VTI (vertical transversely isotropic) acoustic wave equation, Sun [17] found that anisotropy has large influence on the phases and amplitudes of seismic waves and must be considered when processing seismic data. Meléndez-Martínez and Schmitt [18] illustrated that the problems introduced by attempting to use Poisson's ratio to estimate fracture gradient are even further from reality once anisotropy is included. Malehmir and Schmitt $[19,20]$ developed an algorithm to solve reflectivity, transmissivity, velocity, and particle polarization in the case of elastic anisotropy, and found that the tilt of the symmetry planes of anisotropic geological formations will influence observations in AVAz (amplitude versus azimuth) field studies, and knowledge of this tilt becomes essential to fully understand the properties of the subsurface.

In recent years, there has been growing interest in shale anisotropy. However, only few studies have been performed on shale anisotropy's effects on 3D elastic seismic wavefield and quantitative evaluation method. Compared with the acoustic wave, an elastic wave formulation is likely to be a more realistic representation of the behavior of real seismic waves. The quantitative evaluation of anisotropy can identify the observation area where the detectors are less affected by the shale anisotropy. Then in these areas, less affected by anisotropic nature of the substrate, we can apply traditional seismic interpretation and processing method to the selected seismic data, thus improving the accuracy and reliability of sweet spots prediction and reservoir characterization. In this paper, based on the 3D elastic wave equation and the staggered-grid finite-difference method [21,22], the seismic wave forward modeling theory with shale anisotropy was established. Then, by calculating the deviations in waveforms' amplitude and phase which are caused by anisotropy, we developed a quantitative evaluation method. Lastly, we set several models according to the shale reservoir characteristics or the actual oilfield in China, added different levels of anisotropy to them, and applied the proposed method to analyze the shale anisotropy's effects on their seismic wavefield.

\section{Methodology}

\subsection{Forward Modeling for 3D TTI Model}

Considering the shale's strong anisotropy characteristics, the assumption of the isotropic medium is inappropriate, and the adoption of transversely isotropic media is currently recognized as a more reasonable description of shale media $[23,24]$. In our study, the calculation of the seismic wavefield is based on the elastic wave equation:

$$
\rho \frac{\partial^{2} u_{i}}{\partial t^{2}}=c_{i j k l} \frac{\partial^{2} u_{k}}{\partial x_{j} \partial x_{l}}+\rho F_{i}
$$

where $u$ represents the particle's displacement, $\rho$ and $F$ are the medium's density and the external force, respectively, $c_{i j k l}$ is the stiffness matrix in Hooke's law and represents the relationship between stress and strain. Considering the symmetry of stress and elastic coefficient tensors, and assuming that the medium is vertical transversely isotropic (VTI), the stiffness matrix $c_{i j k l}$ can be simplified as:

$$
\left[\begin{array}{cccccc}
c_{11} & c_{12} & c_{13} & 0 & 0 & 0 \\
c_{12} & c_{11} & c_{13} & 0 & 0 & 0 \\
c_{13} & c_{13} & c_{33} & 0 & 0 & 0 \\
0 & 0 & 0 & c_{44} & 0 & 0 \\
0 & 0 & 0 & 0 & c_{44} & 0 \\
0 & 0 & 0 & 0 & 0 & c_{66}
\end{array}\right],
$$


using the weak anisotropy hypothesis proposed by Thomsen [25], the elastic parameters in (2) can be transformed to:

$$
\left\{\begin{array} { c } 
{ \varepsilon = \frac { c _ { 1 1 } - c _ { 3 3 } } { 2 c _ { 3 3 } } , \gamma = \frac { c _ { 6 6 } - c _ { 4 4 } } { 2 c _ { 4 4 } } , } \\
{ v _ { p _ { z } } = \sqrt { \frac { c _ { 3 3 } } { \rho } } , \quad v _ { s _ { z } } = \sqrt { \frac { c _ { 4 4 } } { \rho } } , } \\
{ \delta = \frac { ( c _ { 1 3 } + c _ { 4 4 } ) ^ { 2 } - ( c _ { 3 3 } - c _ { 4 4 } ) ^ { 2 } } { 2 c _ { 3 3 } ( c _ { 3 3 } - c _ { 4 4 } ) } , }
\end{array} \quad \left\{\begin{array}{c}
c_{11}=\rho(1+2 \varepsilon) v_{p_{z^{\prime}}} \\
c_{33}=\rho v_{p_{z^{\prime}}}^{2} \\
c_{44}=\rho v_{s_{z^{\prime}}}^{2} \\
c_{12}=\rho v_{p_{z}}^{2}\left[1+2 \varepsilon-2\left(1-f^{\prime}\right)(1+2 \gamma)\right], \\
c_{13}=\rho v_{p_{z}}^{2} \sqrt{f^{\prime}\left(f^{\prime}+2 \delta\right)}-\rho v_{s_{z^{\prime}}}^{2} \\
c_{66}=\rho(1+2 \gamma) v_{s_{z}}^{2}=0.5\left(c_{11}-c_{12}\right),
\end{array}\right.\right.
$$

where $f^{\prime}=1-v_{s_{z}}^{2} / v_{p_{z}}^{2} \varepsilon$, and $\delta$ represent the difference of P-wave velocity in the direction of vertical, horizontal, and $45^{\circ}$, i.e., the measurement of P-wave anisotropy, $\delta$ represent the anisotropic strength of $\mathrm{S}$-wave, $v_{p_{z}}$ and $v_{s_{z}}$ are the velocity of $\mathrm{P}$ - and S-wave along the vertical (depth) direction, respectively. From Equations (2) and (3), we can see that for the VTI medium, there are six stiffness parameters and five of them are independent with each other.

Equations (1)-(3) establish the elastic wave forward modeling theory of VTI medium (vertical transversely isotropic, i.e., medium with vertical symmetry axis). For TTI medium (tilted transversely isotropic, i.e., medium with tilted symmetry axis), we can make use of its spatial inclination and perform a coordinate transformation to calculate the seismic wavefield.

In this paper, we use the staggered-grid finite-difference method [21,22,26-29] to calculate the elastic wave equation. Details of this method are shown in Appendix A. The finite-difference (FD) method is a crucial numerical tool in the modeling of earthquake ground motion, and the staggered-grid FD algorithm is one of the most popular FD schemes and has been proved to be flexible and relatively accurate in the analysis of wave propagation problems $[27,29,30]$. The staggered-grid FD algorithm computes the pressure at a set of spatial points, and the velocity at another set of spatial points [31]. One of the attractive features of the staggered-grid approach is the velocities are updated independently from the stresses, which allows for a very efficient and concise implementation scheme [27].

\subsection{Quantitative Evaluation of the Shale Anisotropy's Impact}

In order to analyze the shale anisotropy's impact on the seismic wavefield, the comparison between seismic data with and without anisotropy is needed. We use the envelope misfit EM and the phase misfit PM to perform the quantitative evaluation. These two parameters are put forward by Kristek [30] to evaluate both the difference in the amplitude and phase between two signals. Let $S_{R E F}(t)$ be the reference data and $S(t)$ be the data to be compared, the envelope misfit EM is

$$
E M=\frac{\sqrt{\sum_{m}\left[\left|\hat{S}_{R E F}\left(t_{m}\right)\right|-\left|\hat{S}\left(t_{m}\right)\right|\right]^{2}}}{\sqrt{\sum_{m}\left|\hat{S}_{R E F}\left(t_{m}\right)\right|^{2}}}
$$

and the phase misfit PM is

$$
P M=\frac{\sqrt{\sum_{m}\left[\left|\hat{S}_{R E F}\left(t_{m}\right)\right| \cdot \operatorname{Arg}\left(\hat{S}_{R E F}\left(t_{m}\right) / \hat{S}\left(t_{m}\right)\right)\right]^{2}}}{\pi \cdot \sqrt{\sum_{m}\left|\hat{S}_{R E F}\left(t_{m}\right)\right|^{2}}}
$$

where $\hat{S}_{R E F}(t)$ and $\hat{S}(t)$ are the analytical signals of $S_{R E F}(t)$ and $S(t)$, respectively (an analytical signal is a complex-valued function that has no negative frequency components, its real and imaginary parts are real-valued functions related to each other by the Hilbert transform [32]). $|z|$ is the modulus of a complex number $z$ and $\operatorname{Arg}(z)$ is the principal value of $z^{\prime}$ s argument. From Equations (4) and (5), it can be seen that the envelope misfit EM and the phase misfit PM are the relative average errors of two waveforms. If the value of EM is 1, it means that the average amplitude difference between the 
two signals is doubled. For PM, if its value is 1, the polarities of all seismic phases in two signals are completely opposite.

In this paper, we use the following steps (Figure 1) to quantitatively evaluate the impact of shale anisotropy on seismic wavefield:

1. Set the parametric medium model $\mathrm{M}_{0}$ (without anisotropy) according to the geological characteristics of shale reservoirs or the actual oilfield models. Add three sets of different anisotropic parameters to the shale layer of model $\mathrm{M}_{0}$ and establish three different medium models with shale anisotropy, i.e., $\mathrm{M}_{\mathrm{E}}(\varepsilon=0.25, \delta=0), \mathrm{M}_{\mathrm{D}}(\varepsilon=0, \delta=0.25)$, and $\mathrm{M}_{\mathrm{ED}}(\varepsilon=$ $0.25, \delta=0.25)$. The parameters of model $\mathrm{M}_{\mathrm{ED}}$ are reasonably geologically chosen from several shale anisotropy studies in China $([9,17])$, while the other two models $\left(\mathrm{M}_{\mathrm{E}}\right.$ and $\left.\mathrm{M}_{\mathrm{D}}\right)$ are built based on the variable-controlling approach, for the purpose of exploring the impact of different anisotropy parameters;

2. Use the forward modeling method to calculate the elastic wavefields of four medium models $\left(S_{R E F}\right.$ for model $\mathrm{M}_{0}, S_{E}$ for model $\mathrm{M}_{\mathrm{E}}, S_{D}$ for model $\mathrm{M}_{\mathrm{D}}$, and $S_{E D}$ for model $\mathrm{M}_{\mathrm{ED}}$ );

3. Compare the seismic wavefields with each other and calculate the envelope misfit EM and the phase misfit PM of the wavefield $S_{E} / S_{D} / S_{E D}$ from $S_{R E F}$. Evaluate the impact of shale anisotropy on elastic seismic wave response.

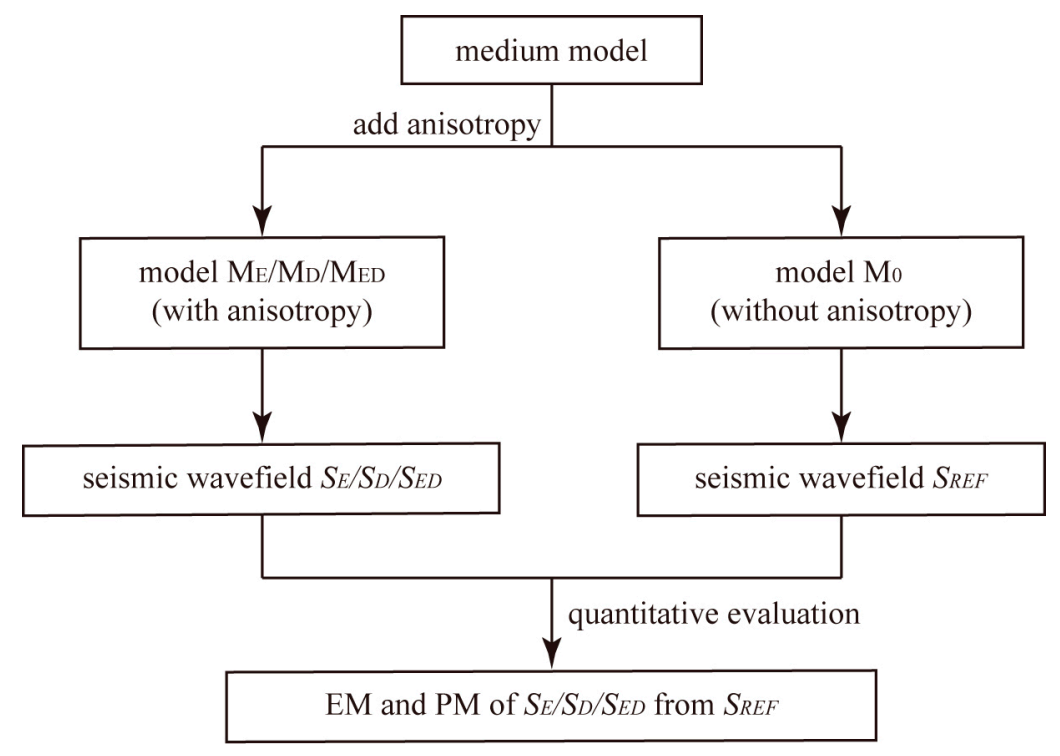

Figure 1. The flow chart of the proposed method for the analysis of anisotropy effects. The procedure of this method starts with adding anisotropy to the medium model, followed by the seismic wavefield forward modeling and quantitative evaluation.

\section{Evaluation of Simulation Models}

In this section, we set two synthetic models of different complexity and used our method to evaluate the impact of anisotropy on their seismic wavefield. Based on the geological sedimentary characteristics of actual shale strata, we set two models, i.e., the horizontal layered VTI model and the curved layered TTI model.

\subsection{Horizontal Layered VTI Model}

\subsubsection{Parameters and Wavefield Data}

The horizontal layered VTI model, seismic source, and observation system are shown in Figure 2. This model has three layers and the shale reservoir is located in the middle. Details of each layer's 
parameters are shown in Table 1. An explosion source is situated in the center of the surface $(1000,1000$, $0 \mathrm{~m}$ ) with the Ricker wavelet time function $(20 \mathrm{~Hz})$. There are two crossing survey lines distributed along the $X$ direction and the $Y$ direction, respectively, each with 201 geophones and $10 \mathrm{~m}$ spacing. The grid number is $201 \times 201 \times 201(\mathrm{X} / \mathrm{Y} / \mathrm{Z})$ with the spatial spacing $10 \mathrm{~m}$ and the time sampling interval is $0.5 \mathrm{~ms}$. According to the steps in Section 2.2 (Figure 1), the horizontal layered VTI model in Figure 2 is set as model $\mathrm{M}_{0}$ (without anisotropy). We added three groups of anisotropic parameters to the shale layer and built up three different anisotropic models, i.e., $\mathrm{M}_{\mathrm{E}}(\varepsilon=0.25, \delta=0), \mathrm{M}_{\mathrm{D}}(\varepsilon=0$, $\delta=0.25)$, and $\mathrm{M}_{\mathrm{ED}}(\varepsilon=0.25, \delta=0.25)$.

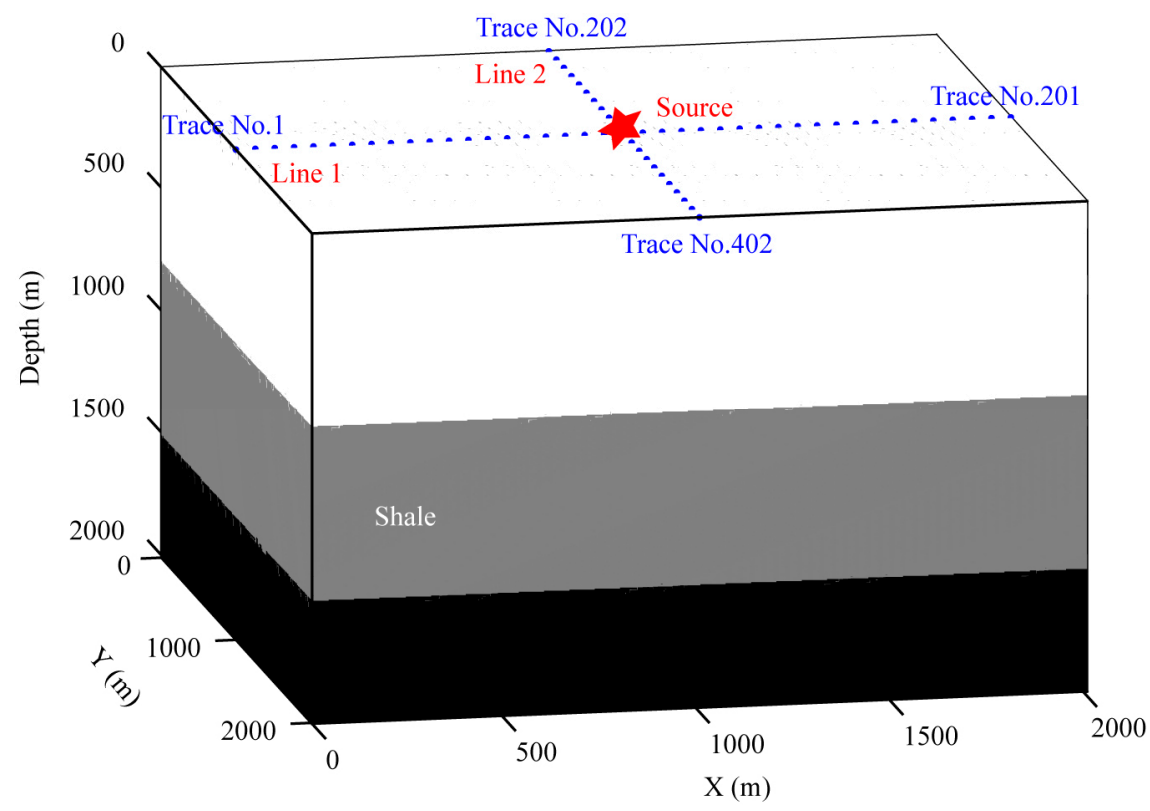

Figure 2. Multi-layered VTI model, source, and observation system. The red star is the source; the blue dots are the detectors distributed on two survey lines (402 geophones in total, with $10 \mathrm{~m}$ spacing).

Table 1. Media parameters for multi-layered VTI model.

\begin{tabular}{ccccc}
\hline Layer & Depth $(\mathbf{m})$ & $\mathbf{V}_{\mathbf{P}}(\mathbf{m} / \mathbf{s})$ & $\mathbf{V}_{\mathbf{S}}(\mathbf{m} / \mathbf{s})$ & Density $\left(\mathbf{g} / \mathbf{c m}^{\mathbf{3}}\right)$ \\
\hline 1 & $0 \sim 800$ & 3000 & 1700 & 2.0 \\
2 & $800 \sim 1500$ & 3500 & 2000 & 2.3 \\
3 & $1500 \sim 2000$ & 4000 & 2300 & 2.4 \\
\hline
\end{tabular}

For models $\mathrm{M}_{0} / \mathrm{M}_{\mathrm{E}} / \mathrm{M}_{\mathrm{D}} / \mathrm{M}_{\mathrm{ED}}$, the seismic waveform records of 402 geophones are respectively synthesized $\left(S_{R E F} / S_{E} / S_{D} / S_{E D}\right)$. The three-component waveforms of model $\mathrm{M}_{0}$, namely $S_{R E F}$, is shown in Figure 3a-c. Comparing the $\mathrm{X}-, \mathrm{Y}-$, and $\mathrm{Z}$-component waveforms of each detector, we can see that the X-and Y-component records of the two survey lines (line 1: No.1 201 and line 2: No.202 402) are different, while the Z-component records are entirely consistent. The reason for this is that the horizontal coordinates of the two survey lines are different (line 1 is arranged along the $X$ direction, and line 2 is along the $Y$ direction). Therefore, for an explosive seismic source, the received $X$ - and Y-waveforms are different, while the Z-component records of two lines are the same, because the symmetry axis of the medium is along the vertical direction (VTI medium). 
(a)
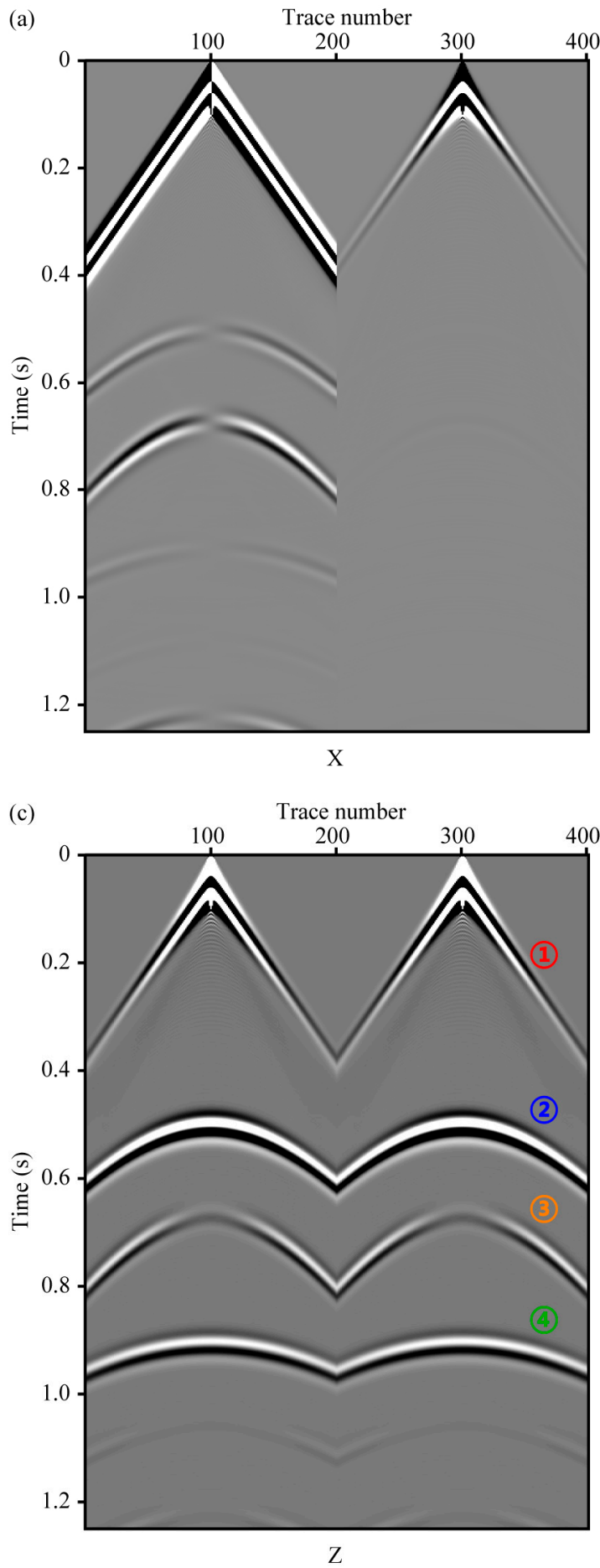

(b)

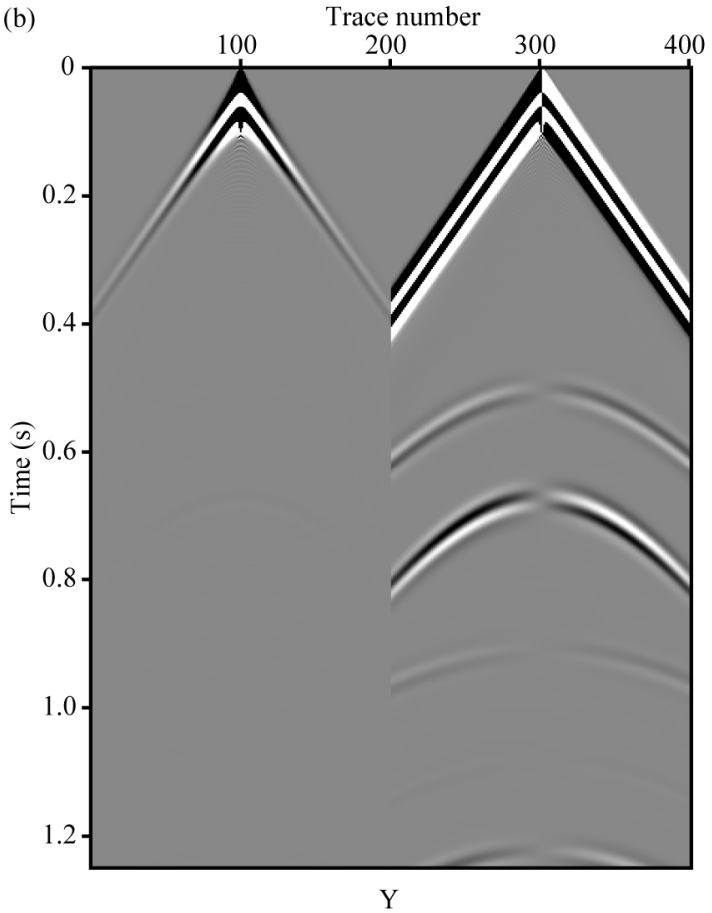

(d)

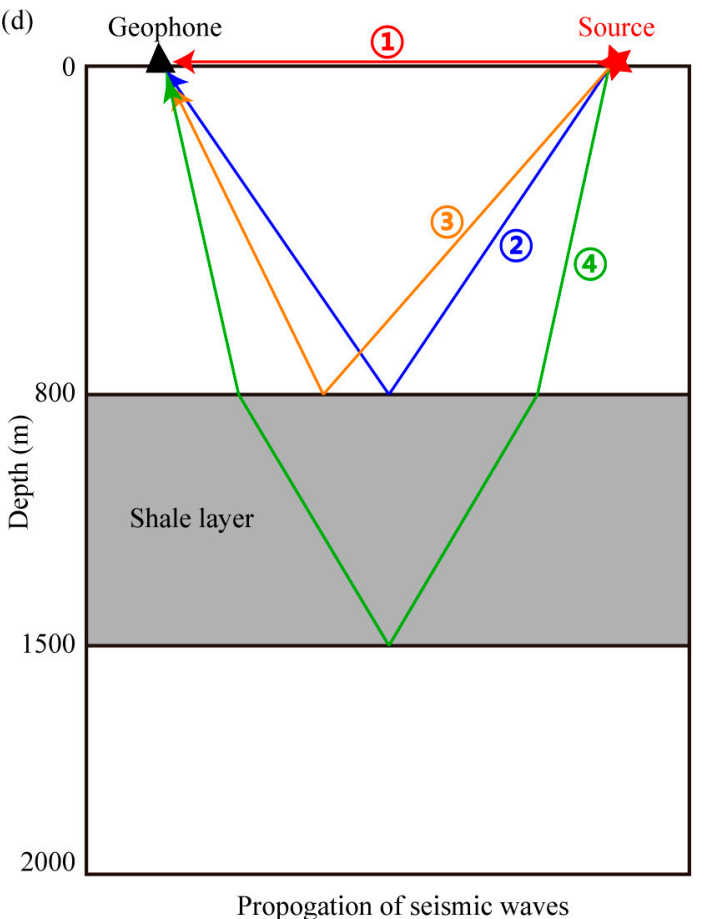

Figure 3. Three-component seismic wavefield of the $\mathrm{M}_{0}$ model and corresponding wave propagation diagrams of four seismic phases. (a)/(b)/(c) are the geophones' $\mathrm{X} / \mathrm{Y} / \mathrm{Z}$ waveform records, respectively, (d) indicates the seismic wave propagation paths corresponding to each phase, with (1) direct $\mathrm{P}$ wave, (2) reflected P-P wave, (3) reflected P-SV wave, and (4) P-P-P-P wave.

Considering that surface seismic exploration usually uses a single-component (generally Z-component) detector, our study mainly focuses on analyzing the influence of shale anisotropy on the Z-component waveforms. It can be seen from Figure $3 \mathrm{c}$ that the Z-component wavefield has four strong phases. Based on the analysis in Figure 3d, these four phases respectively correspond to direct $\mathrm{P}$ wave, reflected P-P wave, reflected P-SV wave, and P-P-P-P wave. 


\subsubsection{Effect of Anisotropy on Different Seismic Phases}

Seismic waves with different phases (direct $\mathrm{P} / \mathrm{S}$, reflected, or transmitted wave) propagate in different paths, so the impact of shale anisotropy on them may also be different. Figure 4 shows the comparison of waveforms from model $\mathrm{M}_{\mathrm{ED}}(\varepsilon=0.25, \delta=0.25)$ and $\mathrm{M}_{0}(\varepsilon=0, \delta=0)$.
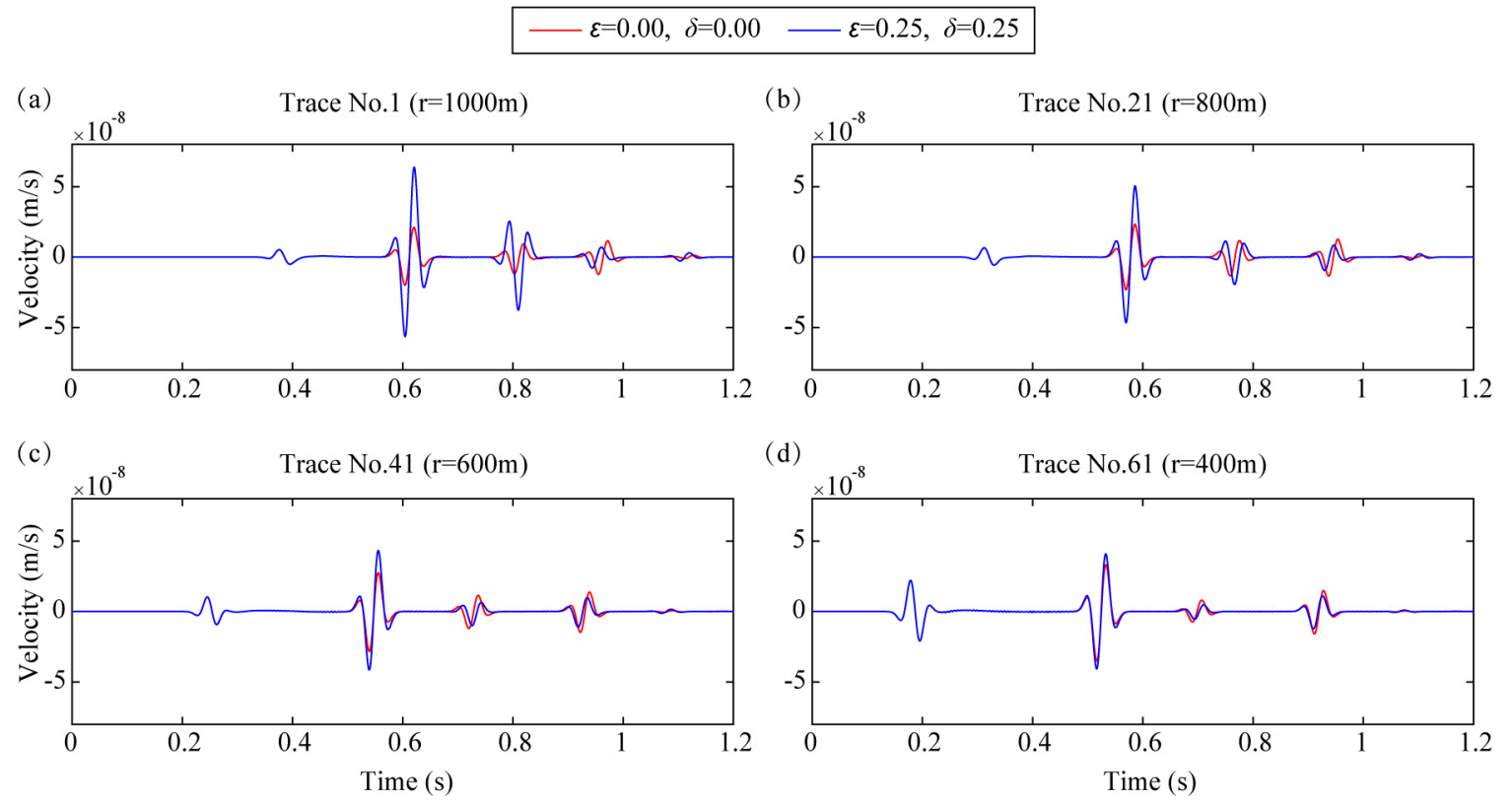

Figure 4. Comparison of Z-component waveforms from model $\mathrm{M}_{\mathrm{ED}}$ and (with/without anisotropy, VTI). $(\mathbf{a}) /(\mathbf{b}) /(\mathbf{c}) /(\mathbf{d})$ are waveforms of geophones No.1/21/41/61 (with epicentral distance 1000/800/600/400 $\mathrm{m}$ ), respectively. Red indicates seismic waveforms of $\mathrm{M}_{0}$ (without anisotropy); blue indicates seismic waveforms from $\mathrm{M}_{\mathrm{ED}}$ (with anisotropy).

From Figure 4, we can find that for each detector:

1. Direct $P$ wave. The first seismic phases (direct $P$ wave) of two models are exactly the same. From its propagation path (Figure 3d), we can see that the direct $P$ wave traveled from the seismic source to the detector through the surface, so it is not affected by the shale anisotropy.

2. Transmitted and reflected P-P-P-P wave. The fourth seismic phases (P-P-P-P wave, its propagation path is shown in Figure 3d) are totally different. When adding anisotropy to the shale layer, the velocities of $\mathrm{P}$-waves along different directions propagating in it are changed and not the same. So this seismic wave's arrival time, amplitude and phase are all influenced by shale anisotropy.

3. Reflected P-P wave and P-SV wave. The arrival time of the second and third phases (reflected P-P and P-SV waves, their paths are shown in Figure 3d) is the same, while their amplitudes are different. The reasons for this are probably because:

- Arrival time. These two waves are both reflected at the interface of the first layer and the shale layer. The first layer is isotropic, so we can use the Snell's law to analyze the reflection:

$$
\frac{v_{P 1}}{\sin \theta_{1}}=\frac{v_{S 1}}{\sin \phi_{1}}
$$

where $v_{P 1}$ is the velocity of incident P-wave (same as the reflected P-wave's velocity) and $v_{S 1}$ is the reflected SV-wave's velocity at the interface, respectively, $\theta_{1}$ and $\phi_{1}$ are the corresponding angles (Figure 5a). When we added anisotropy to the shale layer, velocities of $P$ and $S$ wave in the first layer are not changed, so $v_{P 1}$ and $v_{S 1}$ remain unchanged. From Equation (6), 
we can find that $\theta_{1}, \theta_{2}$, and the propagation paths are not influenced by anisotropy, so is the arrival time.

- Amplitude. The amplitude of reflected P and SV waves can be evaluated using the anisotropic reflectivity and transmissivity calculator code by Malehmir and Schmitt [19]. According to the observation system and source location (Figure 2), the maximum incident angle $\theta_{1}$ of $\mathrm{P}$ wave between the first and second (shale) layer is about $32^{\circ}$. Then, we set $\theta_{1}$ from 0 to $35^{\circ}$ and calculate the reflection coefficients of reflected $\mathrm{P}$ and SV waves. The differences of reflection coefficients with and without anisotropy are shown in Figure 5b. We can find that when adding anisotropy to the VTI model (Figure 2), the reflection coefficients of reflected $\mathrm{P}$ and SV waves are both changed. Considering that the propagation paths of these two waves are unchanged, so the Z-component amplitudes of reflected P-P and P-SV waves are influenced by the shale anisotropy.

(a)

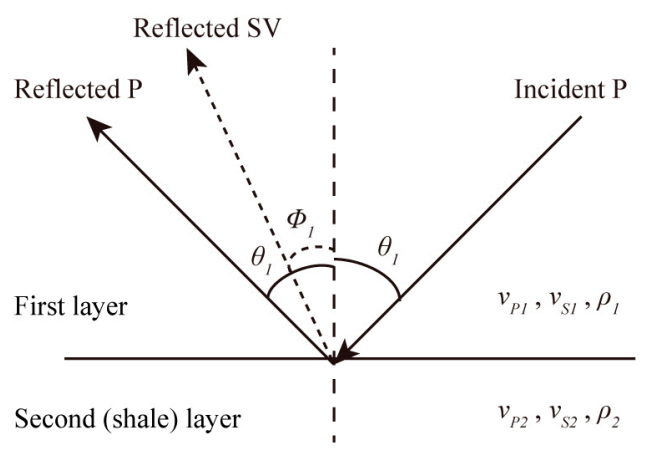

(b)

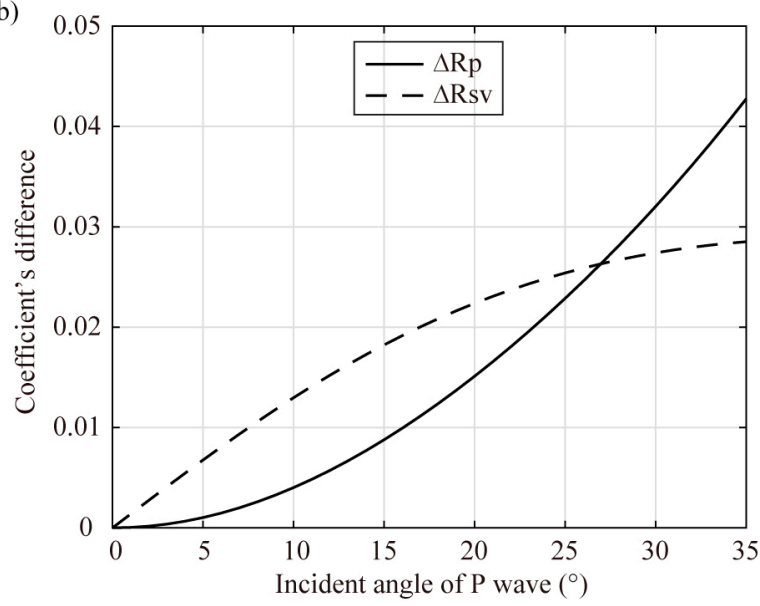

Figure 5. Schematic diagram and coefficients' differences of reflected waves at the interface. (a) Reflection at the interface between first and second (shale) layer in the VTI model (Figure 2). $\theta_{1}$ and $\phi_{1}$ are the angles of $\mathrm{P}$ and SV waves, respectively. (b) Reflection coefficients' differences of models with and without anisotropy $\left(\mathrm{M}_{\mathrm{ED}}\right.$ and $\left.\mathrm{M}_{0}\right)$. Solid and dash line indicates reflected $\mathrm{P}$ and SV waves, respectively.

\subsubsection{Amplitude and Phase Misfit by Anisotropy}

Using the forward modeling method in Section 2.1, we synthesized the seismic waveforms of the four medium models $\mathrm{M}_{0} / \mathrm{M}_{\mathrm{E}} / \mathrm{M}_{\mathrm{D}} / \mathrm{M}_{\mathrm{ED}}(\varepsilon / \delta$ are $0 / 0,0.25 / 0,0 / 0.25$, and $0.25 / 0.25)$. Based on Equations (4) and (5), we calculated the wavefields' amplitude deviation EM and phase deviation PM of the anisotropic model $\mathrm{M}_{\mathrm{E}} / \mathrm{M}_{\mathrm{D}} / \mathrm{M}_{\mathrm{ED}}$ from the model $\mathrm{M}_{0}$ (without anisotropy). The results are shown in Figure 6. Here, the normalized epicentral distance (i.e., epicentral distance divided by wavelength) is used. From Figure 6, we can see the following points:

- The amplitude deviation EM and phase deviation PM of the two survey lines in Z-component are significantly larger than their $\mathrm{X}$ - and Y-components, which indicates that anisotropy has a more significant influence on the vertical component. It is speculated that the reason may be that the propagation distance of the seismic wave in the vertical direction is larger than that in the horizontal direction and the medium model's symmetric axis is along the vertical direction.

- The maximum EM of the two survey lines in Z-component is greater than 1, which indicates that the waveform's amplitude will be significantly affected by the shale anisotropy. In actual seismic exploration, the amplitude is of great importance to the inversion of reservoir parameters, so ignoring anisotropy may lead to errors in reservoir characterization.

- The Z-component phase deviation PM of the two survey lines reaches 0.4. As is mentioned above, if PM is 1 , the polarities of the two signals are completely opposite. So this means that the 
waveforms' phase morphology is also largely changed due to anisotropy. The inaccurate phase may lead to the low resolution of migration imaging results, which also affects the processing and interpretation of actual exploration seismic data.

- The amplitude deviation EM and phase deviation PM of X/Y/Z components on two sides of the source are symmetrical, which is caused by the symmetry axis's verticality of the VTI model. Moreover, the EM all increases with the increase of epicentral distance (offset), while the PM increases first and then decreases. This may because the maximum value of the difference in phase is the odd multiples of $\pi$. Therefore, if the phase's difference gradually increases from 0 to $2 \pi$, the difference in waveform's phase will become bigger first and then smaller, and the calculated PM will also increase first and then decrease correspondingly.

- In most of the results, the deviations of the model $\mathrm{M}_{\mathrm{ED}}(\varepsilon=0.25, \delta=0.25)$ are relatively larger than the other two models $\mathrm{M}_{\mathrm{E}}(\varepsilon=0.25, \delta=0)$ and $\mathrm{M}_{\mathrm{D}}(\varepsilon=0, \delta=0.25)$.

(a)

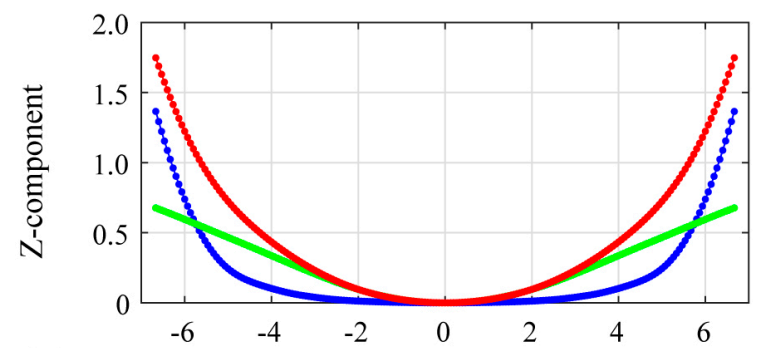

(c)

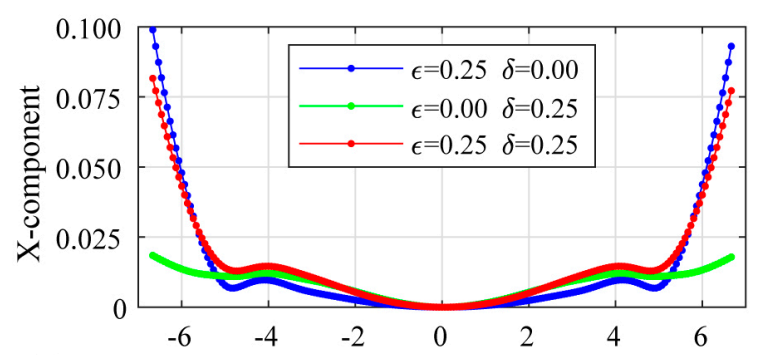

(e)

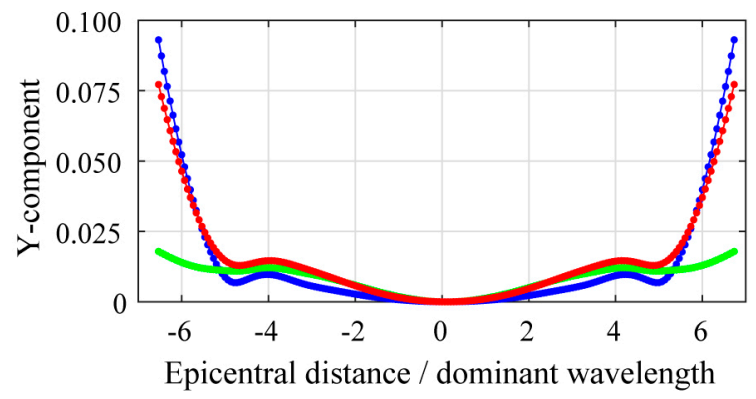

(b)

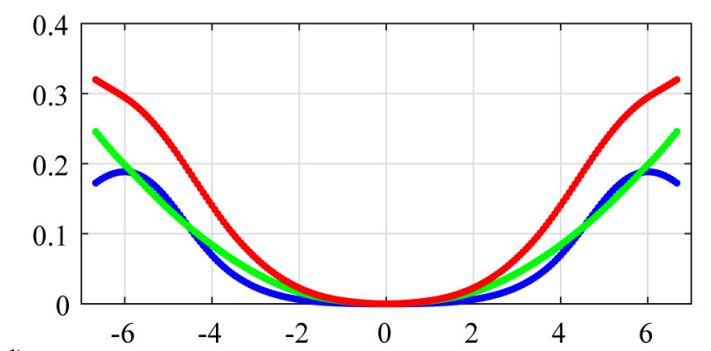

(d)

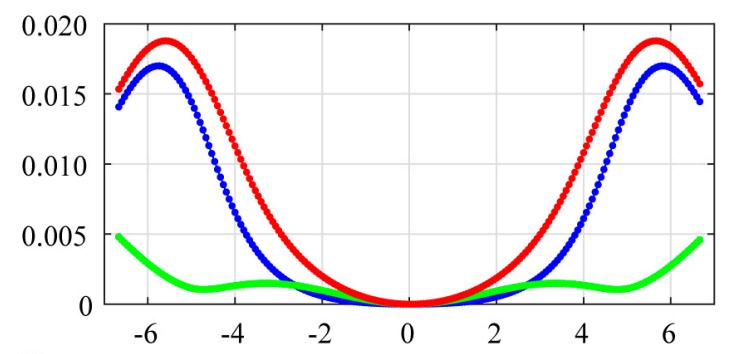

(f)

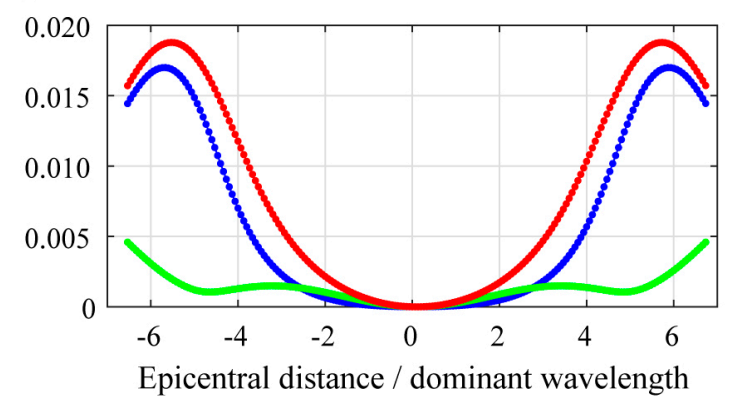

Figure 6. Envelope and phase misfit of detectors' three-component waveforms (VTI model). The blue/green/red lines respectively represent the waveforms' deviation curve of $\mathrm{M}_{\mathrm{E}}(\varepsilon=0.25, \delta=0) / \mathrm{M}_{\mathrm{D}}$ $(\varepsilon=0, \delta=0.25) / \mathrm{M}_{\mathrm{ED}}(\varepsilon=0.25, \delta=0.25)$ with respect to $\mathrm{M}_{0}(\varepsilon=0, \delta=0)$, and the horizontal axis is the normalized epicentral distance (epicentral distance divided by wavelength) of a detector from the seismic source, the sign indicates that the detectors are located on two sides of the source (positive along X/Y axis direction). (a,b) are the envelope and phase misfit recorded by Z-component of line 1, $(\mathbf{c}, \mathbf{d})$ are the $\mathrm{X}$-component's deviations of line 1, (e,f) are the deviations recorded by Y-component of line 2. 


\subsection{Curved Layered TTI Model}

In this section, we analyzed shale anisotropy's impact on the seismic wavefield of a curved multi-layer TTI model [28]. The geometry of the medium model, source, and observation system is shown in Figure 7. Parameters for the wavefields' forward modeling are the same as Section 3.1. Details of each layer's parameters are listed in Table 2 and the shale reservoir is located in the middle. The dip angle of TTI media's symmetry axis is $40^{\circ}$ and the azimuth angle is $0^{\circ}$. Similar to Section 3.1, when anisotropic parameters are added to the shale layer, four models are respectively set: $\mathrm{M}_{0}(\varepsilon=0$, $\delta=0), \mathrm{M}_{\mathrm{E}}(\varepsilon=0.25, \delta=0), \mathrm{M}_{\mathrm{D}}(\varepsilon=0, \delta=0.25)$, and $\mathrm{M}_{\mathrm{ED}}(\varepsilon=0.25, \delta=0.25)$.

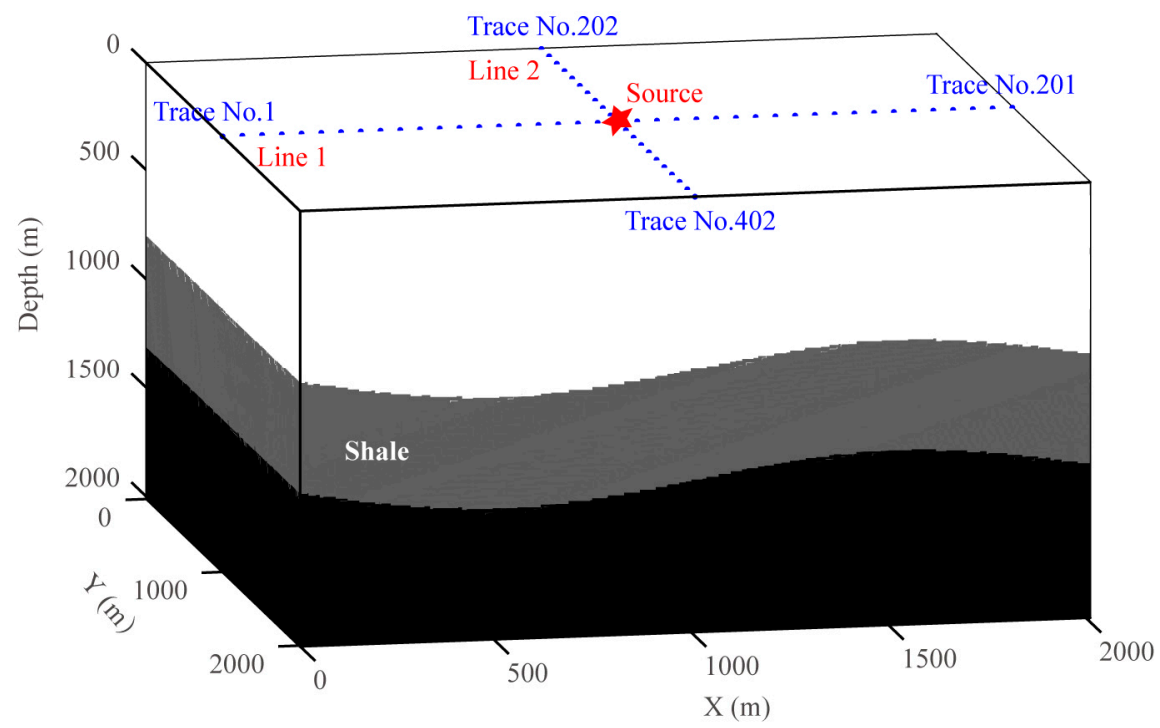

Figure 7. Curved layered TTI model, source, and observation system. The red star is the source; the blue dots are the detectors distributed on two survey lines (402 detectors in total, with $10 \mathrm{~m}$ spacing).

Table 2. Media parameters for multi-layered TTI model.

\begin{tabular}{cccccc}
\hline Layer & Depth $(\mathbf{m})$ & Thickness $(\mathbf{m})$ & $\mathbf{V}_{\mathbf{P}}(\mathbf{m} / \mathbf{s})$ & $\mathbf{V}_{\mathbf{S}}(\mathbf{m} / \mathbf{s})$ & Density $\left(\mathrm{g} / \mathrm{cm}^{\mathbf{3}}\right)$ \\
\hline 1 & 0 & $700-900$ & 3000 & 1700 & 2.0 \\
2 & 800 & 500 & 3500 & 2000 & 2.3 \\
3 & 1500 & $600-800$ & 4000 & 2300 & 2.4 \\
\hline
\end{tabular}

The seismic waveform records of 402 geophones are respectively forward synthesized for four models $\mathrm{M}_{0}, \mathrm{M}_{\mathrm{E}}, \mathrm{M}_{\mathrm{D}}$, and $\mathrm{M}_{\mathrm{ED}}$. The comparison of $\mathrm{Z}$-component wavefields with and without anisotropy in the first survey line (geophone No.1-No.201) is shown in Figure 8, and the waveforms of eight different epicentral distance detectors $(-1000 /-600 /-200 /+200 /+400 /+1000 \mathrm{~m}$, the positive and the negative sign indicate that the detectors are on left and right sides of the seismic source, respectively) are shown in Figure 9. From Figures 8 and 9, it can be seen that before and after anisotropy is added, except the direct $\mathrm{P}$ wave is not affected, the subsequent phases are all affected by different degrees. When adding shale anisotropy, the amplitude of reflected P-P wave (the second phase) is increased. The arrival time, phase, and amplitude of the other phases (P-SV, P-P-P-P, multiple waves, etc.) are all changed due to the shale anisotropy. 

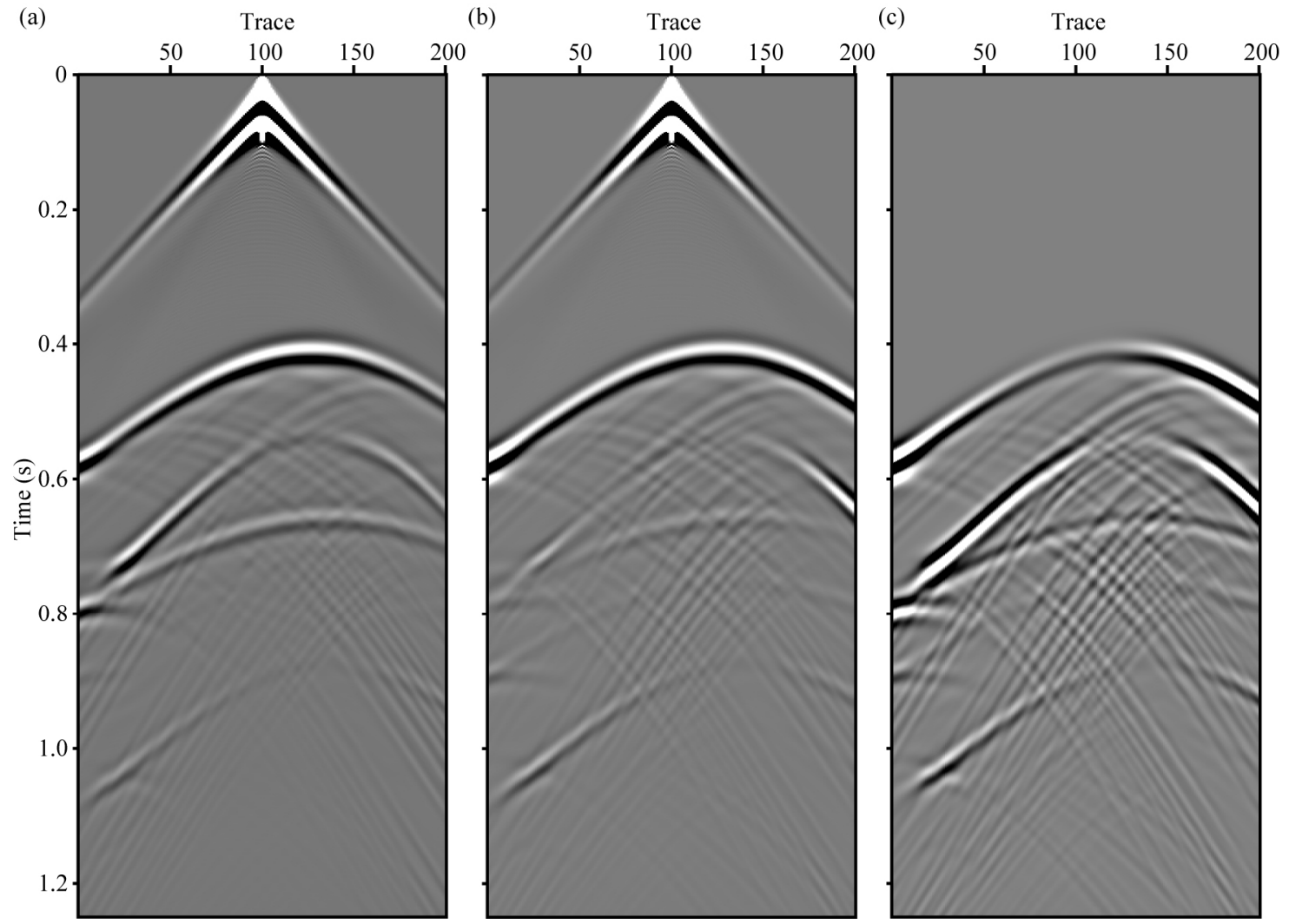

Figure 8. Comparison of Z-component waveforms in line 1 with and without the shale anisotropy (TTI model). (a) is the Z-component wavefield of model $\mathrm{M}_{0}$ (without anisotropy), (b) is the Z-component wavefield of model $\mathrm{M}_{\mathrm{ED}}$ (with anisotropy), and (c) is the residual wavefield for (a) and (b). 

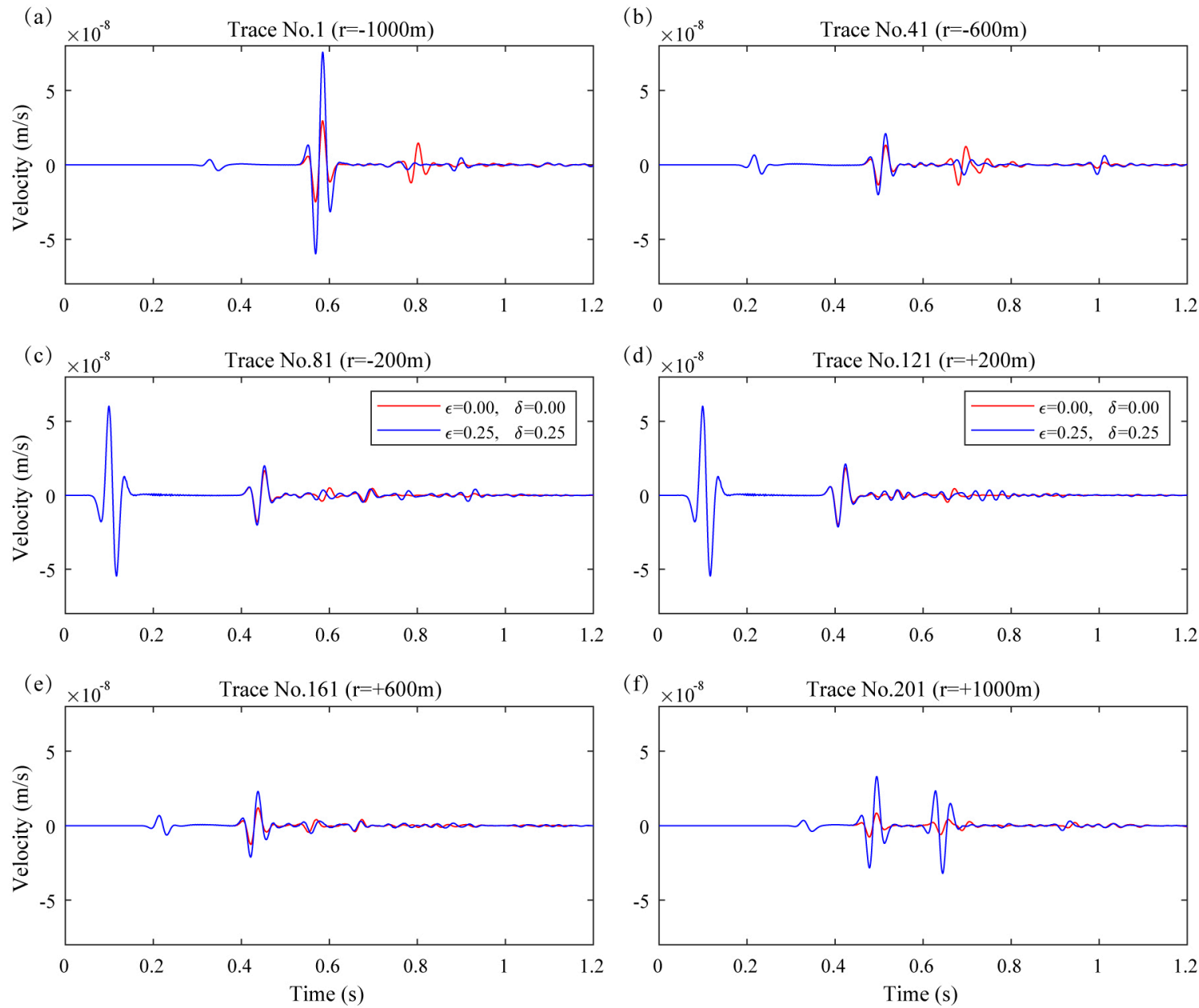

Figure 9. Comparison of Z-component waveforms from model $\mathrm{M}_{\mathrm{ED}}$ and $\mathrm{M}_{0}$ (with/without anisotropy, TTI). (a)-(f) are waveform records of geophones No.1/41/81/121/161/201 (with epicentral distance $-1000 /-600 /-200 /+200 /+600 /+1000 \mathrm{~m}$, the sign indicates that the detectors are located on two sides of the source, positive along $\mathrm{X}$-axis direction). Red indicates synthetic waveforms from the model $\mathrm{M}_{0}$ (without anisotropy); blue indicates seismic waveforms from the model $\mathrm{M}_{\mathrm{ED}}$ (with shale anisotropy).

Similar as Section 3.1, using Equations (4) and (5), we calculated the seismic wavefields' amplitude deviation (envelope misfit EM) and phase deviation (phase misfit PM) of the anisotropic model $\mathrm{M}_{\mathrm{E}} / \mathrm{M}_{\mathrm{D}} / \mathrm{M}_{\mathrm{ED}}(\varepsilon / \delta$ are $0.25 / 0,0 / 0.25,0.25 / 0.25)$ from the model $\mathrm{M}_{0}$ (without anisotropy). The results are shown in Figure 10. Here, the deviation results of $Z$ - and $X$-component are from line 1, while the results of Y-component are from line 2. From Figure 10, we can find that:

- Same as the VTI model, the EM and PM of Z-component are still larger than that of X- and Ycomponents. The maximum EM is still greater than one, and the maximum PM is about 0.5 (relatively large; if PM is 1, the polarities of all phases of the two signals are entirely opposite).

- Unlike the VTI model, with the increase of epicentral distance, the variation trend of EM and PM are complicated. Moreover, the EM and PM of Y-component are symmetrical, while the X- and Zcomponents are not. The reasons are probably because the shape and structure of TTI model are complex, and its symmetry axis is not along the vertical direction. These phenomena prove that the impact of shale anisotropy relies heavily on the model.

- In most of the results, the deviations of models $\mathrm{M}_{\mathrm{ED}}, \mathrm{M}_{\mathrm{E}}$, and $\mathrm{M}_{\mathrm{D}}$ from $\mathrm{M}_{0}$ are close to each other. This indicates that the impact of different anisotropic parameters on the wavefield is complicated in the curved TTI model, and the influence strength of each parameter cannot be determined simply as the horizontal layered VTI model. 
(a)

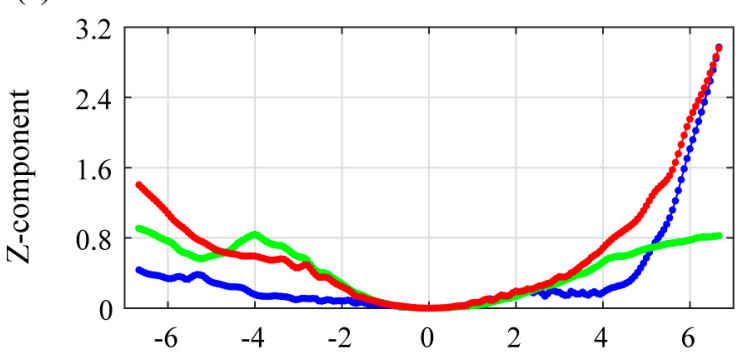

(c)

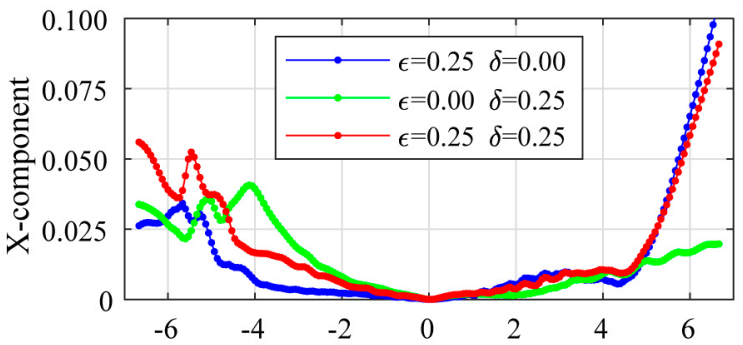

(e)

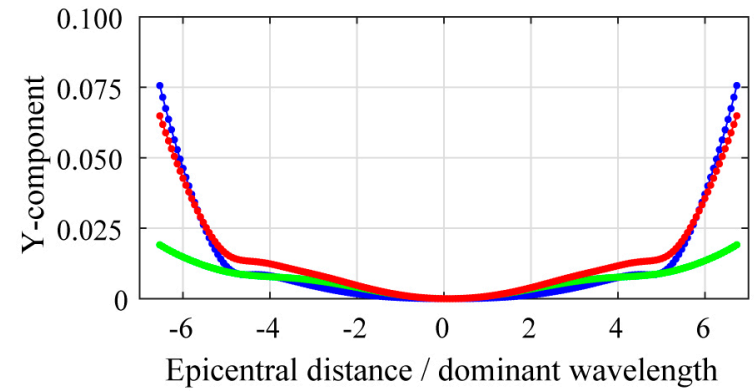

(b)

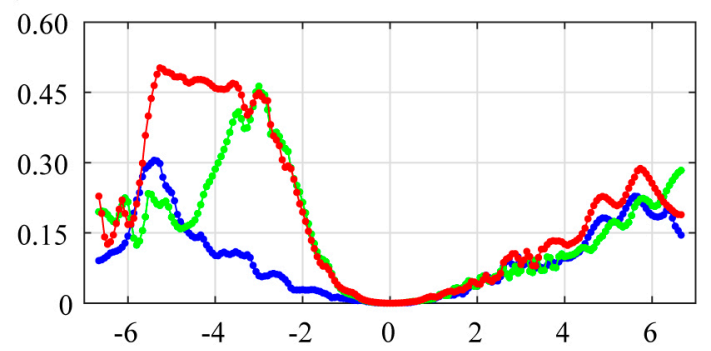

(d)

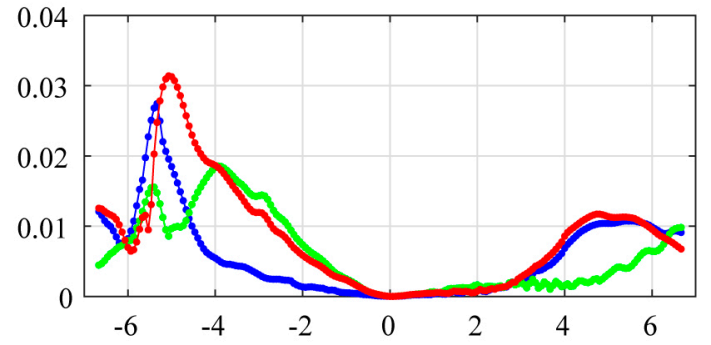

(f)

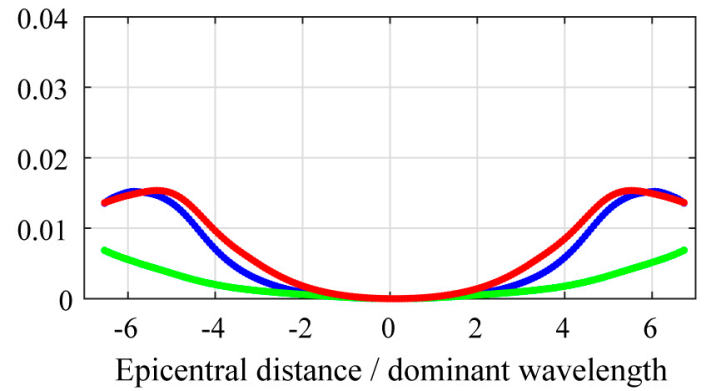

Figure 10. Envelope misfit and phase misfit of detectors' three-component waveforms (TTI model). The meanings of each line and axis are similar to Figure 6.

\section{Evaluation of JY Depression Model}

Through the analysis of the simulation VTI and TTI model, we find that if the medium model is complex (shape is distorted and the symmetry axis is inclined), the influence of shale anisotropy on wavefield is complicated and requires specific analysis. In this section, we try to apply the established evaluation method (Figure 1) to quantitatively analyze the anisotropic influence of an actual shale model (Jiyang depression model) in eastern China from China Petrochemical Corporation (Sinopec).

Figure 11 shows the geometry and velocity parameters of the 3D JY (Jiyang) depression model, and the location of the seismic source and two survey lines (with 402 geophones). When setting the JY depression model, we did some compression along the $\mathrm{X}$ direction and conducted continuation modeling along the $Y$ direction. The shale layer (red) is located in the middle and we add four groups of different anisotropic parameters to it, thus set four models, i.e., $\mathrm{M}_{0}(\varepsilon=0, \delta=0), \mathrm{M}_{\mathrm{E}}(\varepsilon=0.25, \delta=0)$, $\mathrm{M}_{\mathrm{D}}(\varepsilon=0, \delta=0.25)$, and $\mathrm{M}_{\mathrm{ED}}(\varepsilon=0.25, \delta=0.25)$. The grid number of the model is $201 \times 201 \times 201$ with spatial size $10 \times 10 \times 10 \mathrm{~m}$ and the time sampling interval is $0.5 \mathrm{~ms}$. The explosive source is located in the center of the surface $(1000,1000,0 \mathrm{~m})$ and the source time function is Ricker wavelet $(20 \mathrm{~Hz})$.

For four models $\left(\mathrm{M}_{0} / \mathrm{M}_{\mathrm{E}} / \mathrm{M}_{\mathrm{D}} / \mathrm{M}_{\mathrm{ED}}\right.$, with different shale anisotropy), the seismic waveform records of each detector are forward simulated, respectively. Figure 12 shows the comparison of the Z-component wavefield of line 1 (trace numbers No.1-No.201, Figure 11) with and without the shale anisotropy. Figure 13 illustrates the comparison of Z-component waveforms of eight detectors (with different epicentral distances). 


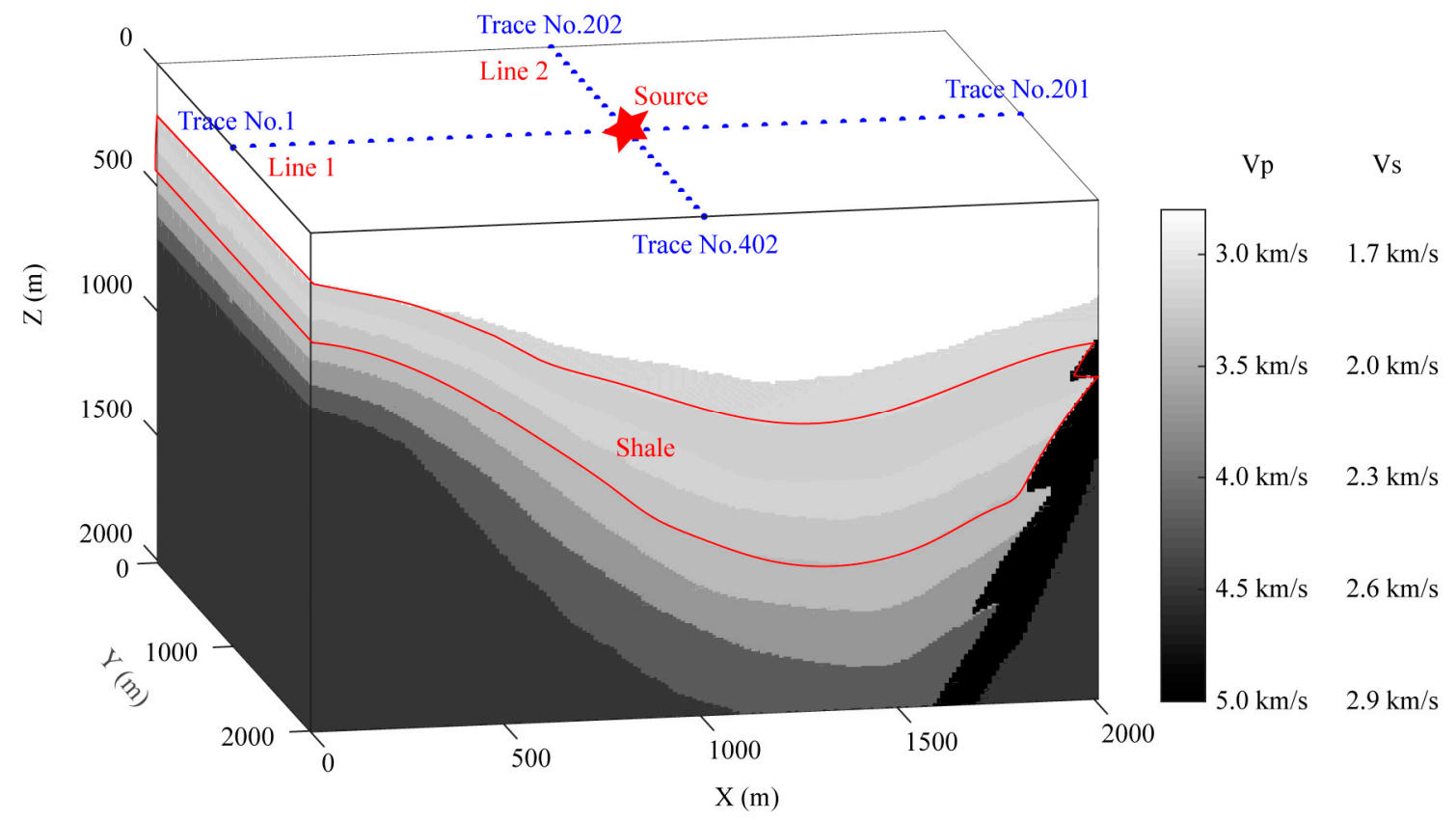

Figure 11. JY depression model, source, and observation system. The red star is the source; the blue dots are the detectors distributed on two survey lines (402 detectors in total, with $10 \mathrm{~m}$ spacing).
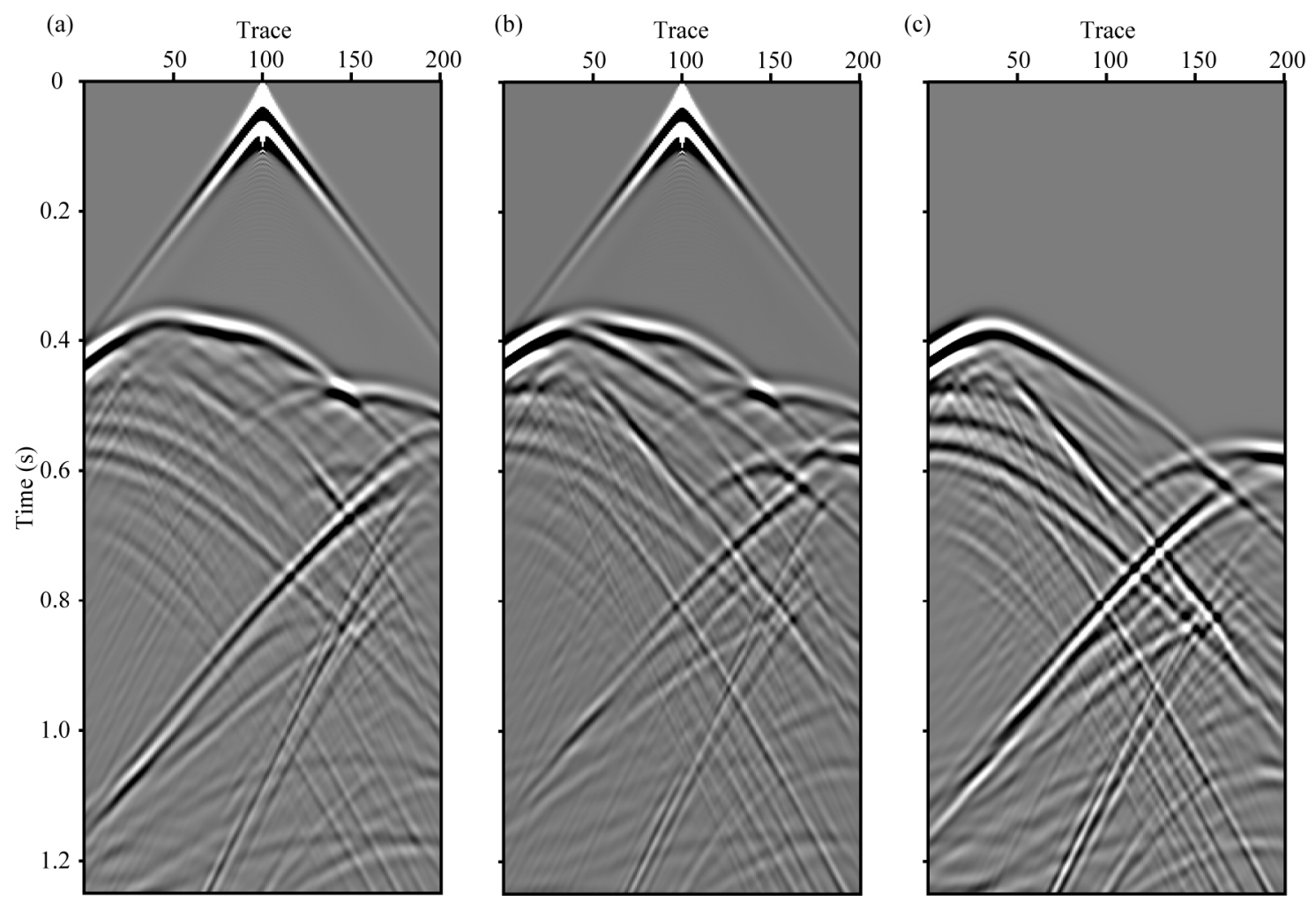

Figure 12. Comparison of Z-component waveforms in line 1 with and without the shale anisotropy (JY model). (a) is the Z-component wavefield of model $\mathrm{M}_{0}$ (without anisotropy), (b) is the Z-component wavefield of model $\mathrm{M}_{\mathrm{ED}}$ (with anisotropy), and (c) is the residual wavefield for (a) and (b). 
(a)
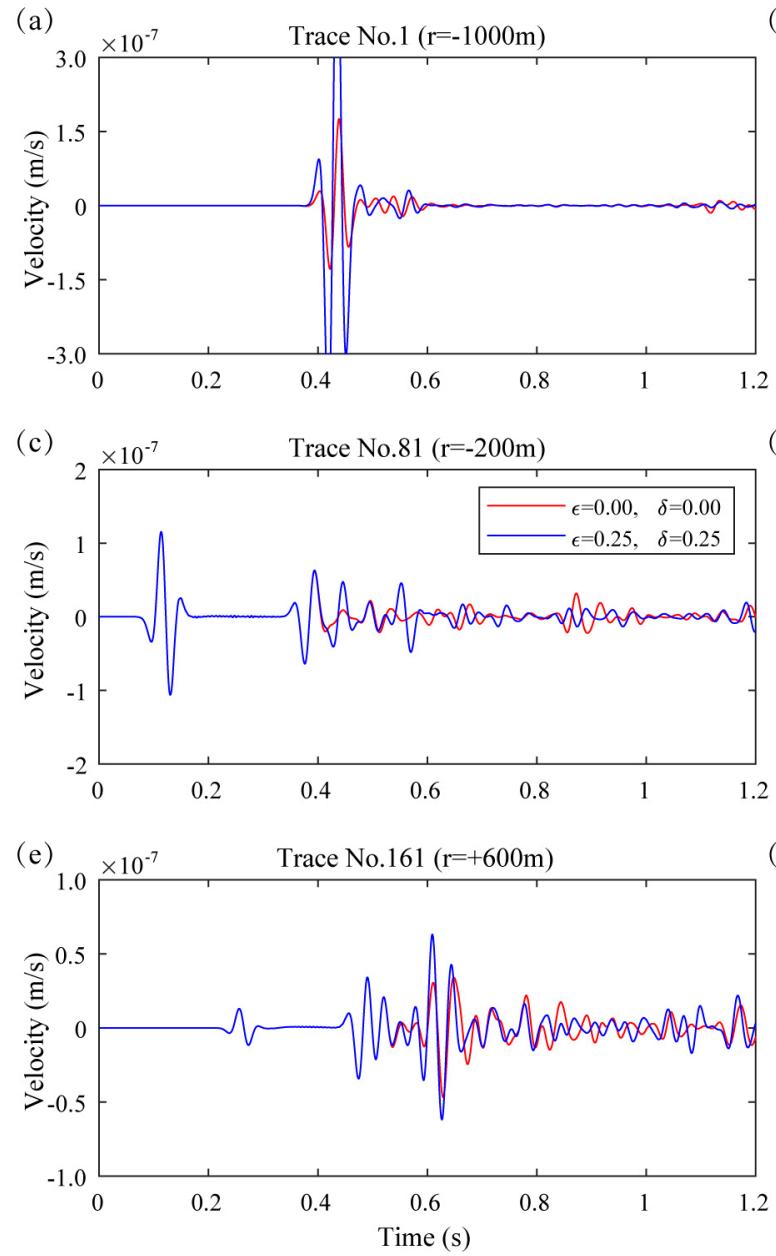

(b)

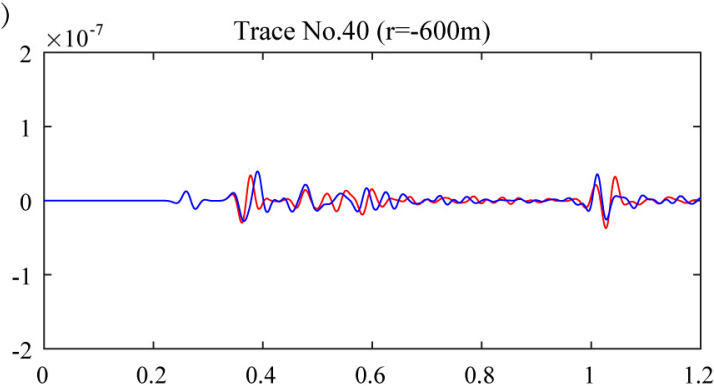

(d)
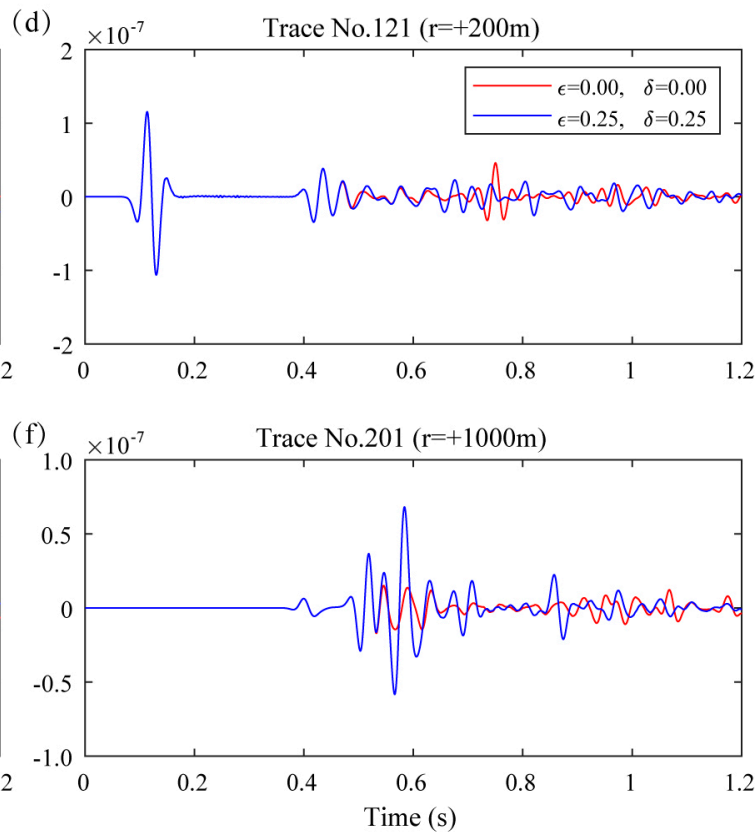

Figure 13. Comparison of Z-component waveforms from model $\mathrm{M}_{\mathrm{ED}}$ and $\mathrm{M}_{0}$ (with/without anisotropy, JY depression model). (a)-(f) are waveform records of geophones No.1/41/81/121/161/201 (with epicentral distance $-1000 /-600 /-200 /+200 /+600 /+1000 \mathrm{~m}$, the sign indicates that the detectors are located on two sides of the source, positive along $\mathrm{X}$-axis direction). Red indicates synthetic waveforms from model $\mathrm{M}_{0}$ (without anisotropy); blue indicates seismic waveforms from model $\mathrm{M}_{\mathrm{ED}}$ (with shale anisotropy).

Since the shape and structure of the JY model are very complex, it is difficult to analyze the impact of each seismic phase accurately. However, from Figures 12 and 13, we can clearly see that when anisotropy is added, in addition to the direct wave, the subsequent seismic phases are all affected and changed. Moreover, comparing the waveforms of detectors on the left and right side of the seismic source (located at trace No.101), it can be seen that their waveforms are not symmetrical and are affected by different degrees.

After the forward modeling of the JY Depression model, similar to Sections 3.1 and 3.2, we analyzed the waveforms' EM and PM of the anisotropic model $\mathrm{M}_{\mathrm{E}} / \mathrm{M}_{\mathrm{D}} / \mathrm{M}_{\mathrm{ED}}$ from the model $\mathrm{M}_{0}$ (without anisotropy). Figure 14 illustrates the calculation results. Here, the deviation results of Z- and Xcomponents are still from the first survey line, while the Y-component results are from the second survey line (Figure 11). From Figure 14, we can find that:

- Similar to VTI and TTI model, the EM and PM of Z-component are still significantly larger than those of the X-and Y- horizontal components. The maximum EM is greater than one and the maximum PM reaches up to 0.5 , which indicates that the impacts of anisotropy on the amplitude and phase are remarkable. 
- Unlike the VTI and TTI model, for the JY model, the variation of EM and PM in the X- and Z-component with the epicentral distance (offset) is complicated, while the EM and PM of Y-component gets bigger with the increase of the epicentral distance.

- $\quad$ Same as TTI model but different from VTI model, for the JY model, the deviations of $\mathrm{M}_{\mathrm{ED}}(\varepsilon=0.25$, $\delta=0.25) / \mathrm{M}_{\mathrm{E}}(\varepsilon=0.25, \delta=0) / \mathrm{M}_{\mathrm{D}}(\varepsilon=0, \delta=0.25)$ are close to each other. This illustrates that the impact of different anisotropic parameters on the wavefield is complicated and needs further study.

(a) Envelope Misfit (EM)

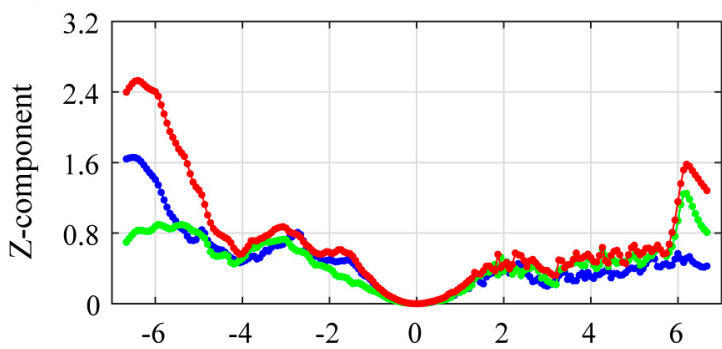

(c)

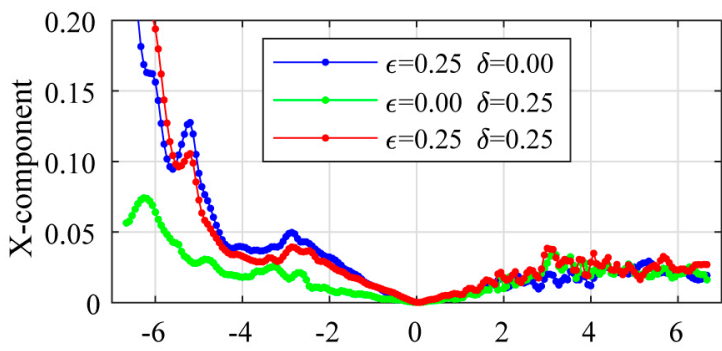

(e)

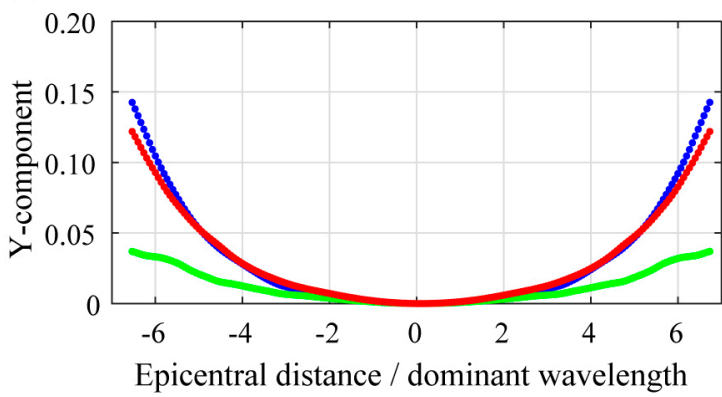

(b) Phase Misfit (PM)

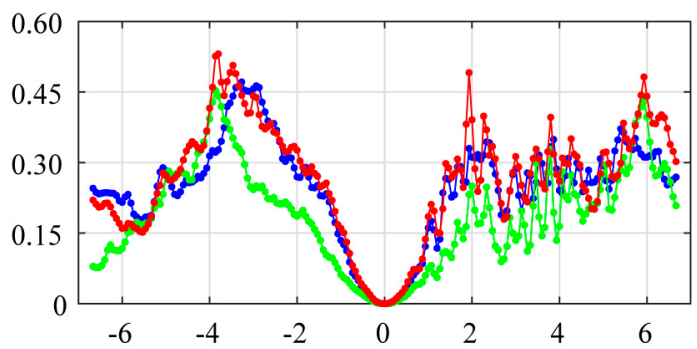

(d)

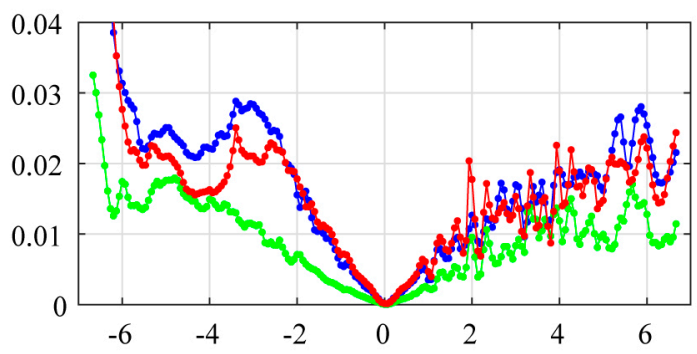

(f)

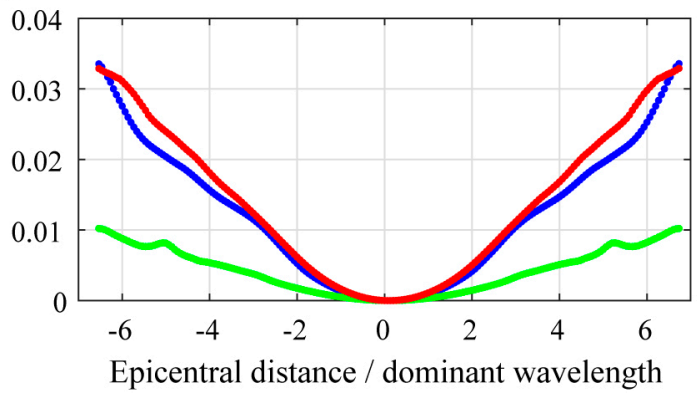

Figure 14. Envelope misfit and phase misfit of detectors' three-component waveforms (JY model). The meanings of each line and axis are similar to Figure 6.

\section{Discussion and Conclusions}

In order to study the impact of shale anisotropy on the seismic wavefield, we proposed a new quantitative evaluation method to calculate the waveform deviations of the anisotropic model from the regular model (without anisotropy). Based on the 3D elastic wave equation and the staggered-grid finite-difference method, the forward modeling theory of the three-component seismic wavefield considering the shale anisotropy was established. Then, we used the envelope misfit (EM) and the phase misfit (PM) to illustrate the differences in waveforms' amplitude and phase morphology caused by anisotropy. Finally, by comparing the waveforms of the models with/without anisotropy and calculating their EM and PM, we can quantitatively evaluate the impact of shale anisotropy on the seismic wavefield.

We applied the proposed method to analyze the anisotropy's effect on two simulation models (horizontal layered VTI model and curved layered TTI model) and one actual oilfield model (JY depression model). Tests on simulation and oilfield models prove that the proposed evaluation method 
is valid and efficient. Moreover, we gained some new cognitions about the anisotropy's effects. (1) The amplitude and phase deviations caused by anisotropy are significant, with EM lager than 1 and PM up to 0.5. Considering that the EM and PM are the relative deviations of two signals, this means that the shale anisotropy may cause the waveforms' amplitudes and phase shapes to be affected by a relatively high level. (2) Anisotropy's impacts on seismic waveform's vertical and horizontal components are different. In most cases, seismic surveys at the surface use the single-component (Z-component) detectors, so during the data interpretation, the Z-component records are adopted. We found that for these three different models, the anisotropy's effect on the Z-component is larger than the other $X / Y$ components. (3) The impact of anisotropy on each detector depends on its offset from the source. When the geophones are set at the surface, for the horizontal layered VTI model, as the offset increases, the EM increases while the PM increases first then decreases. Moreover, the anisotropy's effects on two sides of the seismic source are symmetrical. However, for the curved layered TTI model and JY depression model, the variation trend is not clear. This illustrates that the anisotropy's impact relies heavily on the structure and symmetry axis of the medium model. (4) The influences of different anisotropy parameters on seismic wavefield are different. Based on the variable-controlling approach, we set three different anisotropic models $\left(\mathrm{M}_{\mathrm{E}} / \mathrm{M}_{\mathrm{D}} / \mathrm{M}_{\mathrm{ED}}\right.$, whose $\varepsilon / \delta$ are $0.25 / 0,0 / 0.25$, and $0.25 / 0.25$, respectively). For the VTI model, the deviations of $\mathrm{M}_{\mathrm{ED}}$ from $\mathrm{M}_{0}$ (without anisotropy) are the largest, while for the other two models (TTI and JY model), the deviations of $\mathrm{M}_{\mathrm{E}} / \mathrm{M}_{\mathrm{D}} / \mathrm{M}_{\mathrm{ED}}$ are about at the same level.

According to our research, the impact of shale anisotropy on seismic wavefield is complicated and cannot be simply judged. So, for a particular oilfield model, we need specific analysis. The proposed method provides a useful and quantitative tool for anisotropy's evaluation. Furthermore, by using this method and calculating the waveform misfits from the actual seismic data, we can evaluate the accuracy of different anisotropic models and find the most suitable model for seismic data interpretation. Based on this idea, the anisotropic parameters inversion can be performed by seeking the minimum deviations between the synthetic and the actual seismic wavefield data. Moreover, as detectors with different offsets are affected differently, we can identify the observation area where the detectors are less affected by the shale anisotropy, then use the traditional interpretation method to process the selected wavefield data, so as to improve the accuracy and reliability of sweet spots prediction and reservoir characterization.

Author Contributions: All of the authors have contributed to the present paper. H.L. and X.C. conceived the method and directed the experiments. X.L. and J.L. gave advice for the numerical simulation and provided the model data of the actual oilfield. H.L. and R.W. performed the experiments. H.L. analyzed the data and wrote the paper.

Funding: This research was funded by the National Natural Science Foundation of China, grant number 41804050, 41974156, U1663207, and the State Energy Center for Shale Oil Research and Development, Sinopec, grant number G5800-18-ZS-KFNY002.

Conflicts of Interest: The authors declare no conflict of interest.

\section{Appendix A. Staggered-Grid Finite-Difference Algorithm}

In this paper, we used the staggered-grid finite-difference algorithm [21,22,26-28] to compute the seismic wavefield. This section gives a detailed introduction on this method. The 3D elastic wave equation can be written as:

$$
\rho(x) \frac{\partial v_{i}}{\partial t}(x, t)=\sum_{j=1}^{3} \frac{\partial T_{i j}}{\partial x_{j}}(x, t)+\rho(x) F_{i}(x, t),
$$


where $x$ and $t$ are the space and time variables, subscript $1 / 2 / 3$ refers to the $X / Y / Z$ direction of space. $\rho(x)$ is the density and $v_{i}(x, t)$ is the particle velocity, $T_{i j}(x, t)$ is the stress tensor, and $F_{i}(x, t)$ is the external force. For the VTI (vertical transversely isotropic) medium:

$$
\left[\begin{array}{c}
\frac{\partial T_{11}}{\partial t} \\
\frac{\partial T_{22}}{\partial t} \\
\frac{\partial T_{33}}{\partial t} \\
\frac{\partial T_{23}}{\partial t} \\
\frac{\partial T_{13}}{\partial t} \\
\frac{\partial T_{12}}{\partial t}
\end{array}\right]=\left[\begin{array}{cccccc}
c_{11} & c_{12} & c_{13} & 0 & 0 & 0 \\
c_{12} & c_{11} & c_{13} & 0 & 0 & 0 \\
c_{13} & c_{13} & c_{33} & 0 & 0 & 0 \\
0 & 0 & 0 & c_{44} & 0 & 0 \\
0 & 0 & 0 & 0 & c_{44} & 0 \\
0 & 0 & 0 & 0 & 0 & c_{66}
\end{array}\right]\left[\begin{array}{c}
\frac{\partial v_{1}}{\partial x_{1}} \\
\frac{\partial v_{2}}{\partial x_{2}} \\
\frac{\partial v_{3}}{\partial x_{3}} \\
\frac{\partial v_{2}}{\partial x_{3}}+\frac{\partial v_{3}}{\partial x_{2}} \\
\frac{\partial v_{1}}{\partial x_{3}}+\frac{\partial v_{3}}{\partial x_{1}} \\
\frac{\partial v_{1}}{\partial x_{2}}+\frac{\partial v_{2}}{\partial x_{1}}
\end{array}\right],
$$

in which $c_{i j k l}$ is the stiffness matrix and can be represented by $v_{p_{z}} / v_{s_{z}} / \varepsilon / \delta / \gamma$ (see Equation (2) and Section 2.1). The staggered-grid finite-difference algorithm is built based on the following principle in order to approximate partial derivatives:

$$
\frac{\partial f}{\partial a}(a) \approx \frac{1}{\Delta a} \sum_{m=1}^{N} k_{m} \times\left[f\left(a+\frac{2 m-1}{2} \Delta a\right)-f\left(a-\frac{2 m-1}{2} \Delta a\right)\right],
$$

where $k_{m}$ is the finite difference coefficient (calculated by solving the Vandermonde matrix [33]) and $\mathrm{N}$ is the order number (we use the 5-th order during the forward modeling in this paper).

Following Equation (A3), the displacement velocity in Equation (A1) yields the following approximations (here shows $v_{1}$ for example):

$$
\begin{aligned}
& v_{1}\left(x_{1}, x_{2}, x_{3}, t+\Delta t\right)=v_{1}\left(x_{1}, x_{2}, x_{3}, t\right)+F_{1} \Delta t+ \\
& \frac{\Delta t}{\Delta x} \frac{1}{\rho} \sum_{m=1}^{N} k_{m}\left[T_{11}\left(x_{1}+\frac{2 m-1}{2} \Delta x, x_{2}, x_{3}, t+\frac{1}{2} \Delta t\right)-T_{11}\left(x_{1}-\frac{2 m-1}{2} \Delta x, x_{2}, x_{3}, t+\frac{1}{2} \Delta t\right)+\right. \\
& T_{12}\left(x_{1}, x_{2}+\frac{2 m-1}{2} \Delta x, x_{3}, t+\frac{1}{2} \Delta t\right)-T_{12}\left(x_{1}, x_{2}-\frac{2 m-1}{2} \Delta x, x_{3}, t+\frac{1}{2} \Delta t\right)+ \\
& \left.T_{13}\left(x_{1}, x_{2}, x_{3}+\frac{2 m-1}{2} \Delta x, t+\frac{1}{2} \Delta t\right)-T_{13}\left(x_{1}, x_{2}, x_{3}-\frac{2 m-1}{2} \Delta x, t+\frac{1}{2} \Delta t\right)\right]
\end{aligned}
$$

Following Equation (A3), the stress tensor in Equation (A2) yields the following approximations (here shows $T_{11}$ for example):

$$
\begin{aligned}
& T_{11}\left(x_{1}, x_{2}, x_{3}, t+\Delta t\right)=T_{11}\left(x_{1}, x_{2}, x_{3}, t\right)+ \\
& \frac{\Delta t}{\Delta x} \sum_{m=1}^{N} k_{m}\left[c_{11}\left[v_{1}\left(x_{1}+\frac{2 m-1}{2} \Delta x, x_{2}, x_{3}, t+\frac{1}{2} \Delta t\right)-v_{11}\left(x_{1}-\frac{2 m-1}{2} \Delta x, x_{2}, x_{3}, t+\frac{1}{2} \Delta t\right)\right]+\right. \\
& c_{12}\left[v_{2}\left(x_{1}, x_{2}+\frac{2 m-1}{2} \Delta x, x_{3}, t+\frac{1}{2} \Delta t\right)-v_{2}\left(x_{1}, x_{2}-\frac{2 m-1}{2} \Delta x, x_{3}, t+\frac{1}{2} \Delta t\right)\right]+ \\
& \left.c_{13}\left[v_{3}\left(x_{1}, x_{2}, x_{3}+\frac{2 m-1}{2} \Delta x, t+\frac{1}{2} \Delta t\right)-v_{3}\left(x_{1}, x_{2}, x_{3}-\frac{2 m-1}{2} \Delta x, t+\frac{1}{2} \Delta t\right)\right]\right]
\end{aligned}
$$

in Equations (A4) and (A5), $\Delta x$ and $\Delta t$ are the spatial and time steps to approximate the spatial and time derivatives. For the staggered-grid finite-difference algorithm, the velocity and stress fields are staggered in time and space (Figure A1, [21,27,34]).

During the wavefield forward modeling, $\Delta x$ and $\Delta t$ should be chosen carefully in order to meet the stability condition:

$$
\Delta t \leq \frac{1}{\sqrt{3}} \cdot \frac{\Delta x}{v_{\max }}, \Delta x \leq \frac{\lambda_{\min }}{5}
$$

where $v_{\max }$ is the largest velocity in the medium and $\lambda_{\min }$ is the minimum wavelength of seismic wavefield. Moreover, when dealing with the boundary, we used the absorption boundary condition, i.e., applied the following attenuation function to the boundary area:

$$
A(i)=\exp \left[-a *(i / N)^{2}\right]
$$


in which $N$ and $i$ are the total number and the serial number of grid points in the attenuation area, $a$ is the attenuation coefficient (in this paper, $N=100, a=0.1$ ).

(a)

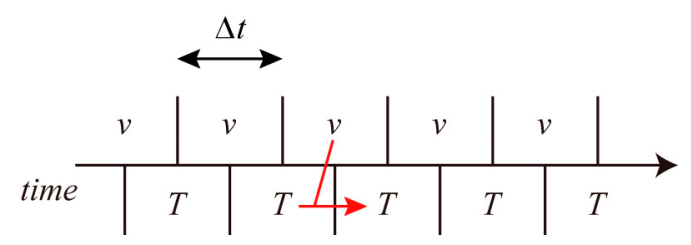

(b)

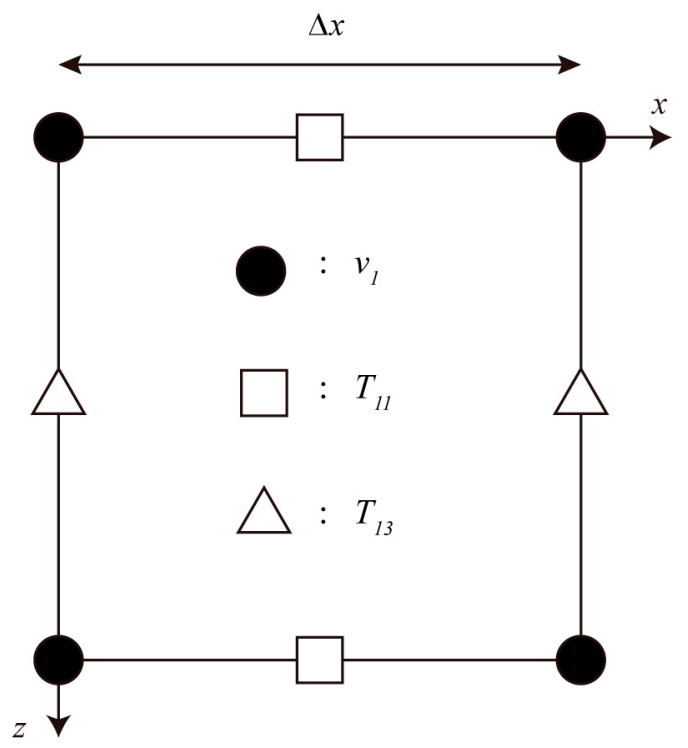

Figure A1. Schematic diagram of the staggered-grid finite-difference method. (a) Staggered grids in time; (b) staggered grids in space, $v$ is the velocity and $T$ is the stress, subscript 1 and 3 refer to the $X$ and $\mathrm{Z}$ direction of space.

\section{References}

1. Zahid, S.; Bhatti, A.A.; Ahmad Khan, H.; Ahmad, T. Development of Unconventional Gas Resources: Stimulation Perspective. In Proceedings of the Production and Operations Symposium, Oklahoma City, OK, USA, 31 March-3 April 2007; Society of Petroleum Engineers: Dallas, TX, USA, 2007.

2. Jia, C.; Zheng, M.; Zhang, Y. Unconventional hydrocarbon resources in China and the prospect of exploration and development. Pet. Explor. Dev. 2012, 39, 139-146. [CrossRef]

3. Stark, P.H.; Chew, K.; Fryklund, B. The role of unconventional hydrocarbon resources in shaping the energy future. In Proceedings of the IPTC 2007 International Petroleum Technology Conference, Dubai, UAE, 4-6 December 2007; Volume 3, pp. 1876-1881.

4. Prasad, M.; Pal-Bathija, A.; Johnston, M.; Rydzy, M.; Batzle, M. Rock physics of the unconventional. Lead. Edge 2009, 28, 34-38. [CrossRef]

5. Vernik, L.; Nur, A. Ultrasonic velocity and anisotropy of hydrocarbon source rocks. Geophysics 1992, 57, 727-735. [CrossRef]

6. Johnston, J.E.; Christensen, N.I. Seismic anisotropy of shales. J. Geophys. Res. Solid Earth 1995, 100, 5991-6003. [CrossRef]

7. Grechka, V.; Yaskevich, S. Azimuthal anisotropy in microseismic monitoring: A Bakken case study. Geophysics 2014, 79, KS1-KS12. [CrossRef]

8. Ong, O.N.; Schmitt, D.R.; Kofman, R.S.; Haug, K. Static and dynamic pressure sensitivity anisotropy of a calcareous shale. Geophys. Prospect. 2016, 64, 875-897. [CrossRef]

9. Liu, X.-W.; Guo, Z.-Q.; Liu, C.; Liu, Y.-W. Anisotropy rock physics model for the Longmaxi shale gas reservoir, Sichuan Basin, China. Appl. Geophys. 2017, 14, 21-30. [CrossRef]

10. Wang, Y.; Li, C.H. Investigation of the P- and S-wave velocity anisotropy of a Longmaxi formation shale by real-time ultrasonic and mechanical experiments under uniaxial deformation. J. Pet. Sci. Eng. 2017, 158, 253-267. [CrossRef]

11. Zhang, F.; Li, X.; Qian, K. Estimation of anisotropy parameters for shale based on an improved rock physics model, part 1: Theory. J. Geophys. Eng. 2017, 14, 143-158. [CrossRef]

12. Zhang, Y.Y.; Jin, Z.J.; Chen, Y.Q.; Liu, X.W.; Han, L.; Jin, W.J. Pre-stack seismic density inversion in marine shale reservoirs in the southern Jiaoshiba area, Sichuan Basin, China. Pet. Sci. 2018, 15, 484-497. [CrossRef] 
13. Carcione, J.M.; Helle, H.B.; Zhao, T. Effects of attenuation and anisotropy on reflection amplitude versus offset. Geophysics 1998, 63, 1652-1658. [CrossRef]

14. King, A.; Talebi, S. Anisotropy Effects on Microseismic Event Location. Pure Appl. Geophys. 2007, 164, 2141-2156. [CrossRef]

15. Warpinski, N.R.; Waltman, C.K.; Du, J.; Ma, Q. Anisotropy Effects in Microseismic Monitoring. In Proceedings of the SPE Annual Technical Conference and Exhibition, New Orleans, LA, USA, 4-7 October 2009; Society of Petroleum Engineers: Dallas, TX, USA, 2009.

16. Tsvankin, I.; Gaiser, J.; Grechka, V.; van der Baan, M.; Thomsen, L. Seismic anisotropy in exploration and reservoir characterization: An overview. Geophysics 2010, 75, 75A15-75A29. [CrossRef]

17. Sun, W.; Fu, L.; Guan, X.; Wei, W. A study on anisotropy of shale using seismic forward modeling in shale gas exploration. Chin. J. Geophys. 2013, 56, 961-970.

18. Meléndez-Martínez, J.; Schmitt, D.R. A comparative study of the anisotropic dynamic and static elastic moduli of unconventional reservoir shales: Implication for geomechanical investigations. Geophysics 2016, 81, D253-D269. [CrossRef]

19. Malehmir, R.; Schmitt, D.R. ARTc: Anisotropic reflectivity and transmissivity calculator. Comput. Geosci. 2016, 93, 114-126. [CrossRef]

20. Malehmir, R.; Schmitt, D.R. Acoustic Reflectivity From Variously Oriented Orthorhombic Media: Analogies to Seismic Responses From a Fractured Anisotropic Crust. J. Geophys. Res. Solid Earth 2017, 122, 10069-10085. [CrossRef]

21. Virieux, J. SH-wave propagation in heterogeneous media: Velocity-stress finite-difference method. Geophysics 1984, 49, 1933-1942. [CrossRef]

22. Virieux, J. P-SV wave propagation in heterogeneous media: Velocity-stress finite-difference method. Geophysics 1986, 51, 889-901. [CrossRef]

23. Qin, Y.; Zhang, Z.; Li, S. CDP mapping in tilted transversely isotropic (TTI) media. Part I: Method and effectiveness. Geophys. Prospect. 2003, 51, 315-324.

24. Guo, P.; McMechan, G.A. Sensitivity of 3D 3C synthetic seismograms to anisotropic attenuation and velocity in reservoir models. Geophysics 2017, 82, T79-T95. [CrossRef]

25. Thomsen, L. Weak elastic anisotropy. Geophysics 1986, 51, 1954-1966. [CrossRef]

26. Tsingas, C.; Vafidis, A.; Kanasewich, E.R. Elastic Wave Propagation in Transversely Isotropic Media Using Finite Differences. Geophys. Prospect. 1990, 38, 933-949. [CrossRef]

27. Graves, R.W. Simulating seismic wave propagation in 3D elastic media using staggered-grid finite differences. Bull. Seismol. Soc. Am. 1996, 86, 1091-1106.

28. Okaya, D.A.; McEvilly, T.V. Elastic wave propagation in anisotropic crustal material possessing arbitrary internal tilt. Geophys. J. Int. 2003, 153, 344-358. [CrossRef]

29. Wang, L.; Chang, X.; Wang, Y. Forward modeling of pseudo P waves in TTI medium using staggered grid. Chin. J. Geophys. 2016, 59, 1046-1058.

30. Kristek, J.; Moczo, P.; Archuleta, R.J. Efficient Methods to Simulate Planar Free Surface in the 3D 4th-Order Staggered-Grid Finite-Difference Schemes. Stud. Geophys. Geod. 2002, 46, 355-381. [CrossRef]

31. Danggo, M.Y.; Mungkasi, S. A staggered grid finite difference method for solving the elastic wave equations. J. Phys. Conf. Ser. 2017, 909, 0-5. [CrossRef]

32. Smith, J.O., III. Mathematics of the Discrete Fourier Transform (DFT): With Audio Applications, 2nd ed.; W3K Publishing: Stanford, CA, USA, 2007; ISBN 978-0974560748.

33. Liu, Y.; Sen, M.K. An implicit staggered-grid finite-difference method for seismic modelling. Geophys. J. Int. 2009, 179, 459-474. [CrossRef]

34. Bossy, E.; Talmant, M.; Laugier, P. Three-dimensional simulations of ultrasonic axial transmission velocity measurement on cortical bone models. J. Acoust. Soc. Am. 2004, 115, 2314-2324. [CrossRef]

(C) 2019 by the authors. Licensee MDPI, Basel, Switzerland. This article is an open access article distributed under the terms and conditions of the Creative Commons Attribution (CC BY) license (http://creativecommons.org/licenses/by/4.0/). 